\title{
The Dynamic Spread of the Forward CDS with General Random Loss
}

\author{
Kun Tian, ${ }^{1}$ Dewen Xiong, ${ }^{1}$ and Zhongxing Ye ${ }^{1,2}$ \\ ${ }^{1}$ Department of Mathematics, Shanghai Jiao Tong University, Shanghai 200240, China \\ ${ }^{2}$ School of Business Information, Shanghai University of International Business and Economics, Shanghai 201620, China
}

Correspondence should be addressed to Kun Tian; tkrp1980@sjtu.edu.cn

Received 24 February 2014; Accepted 3 May 2014; Published 27 May 2014

Academic Editor: Igor Leite Freire

Copyright (C) 2014 Kun Tian et al. This is an open access article distributed under the Creative Commons Attribution License, which permits unrestricted use, distribution, and reproduction in any medium, provided the original work is properly cited.

\begin{abstract}
We assume that the filtration $\mathbb{F}$ is generated by a $d$-dimensional Brownian motion $W=\left(W_{1}, \ldots, W_{d}\right)^{\prime}$ as well as an integer-valued random measure $\mu(d u, d y)$. The random variable $\widetilde{\tau}$ is the default time and $L$ is the default loss. Let $\mathbb{G}=\left\{\mathscr{G}_{t} ; t \geq 0\right\}$ be the progressive enlargement of $\mathbb{F}$ by $(\widetilde{\tau}, L)$; that is, $\mathbb{G}$ is the smallest filtration including $\mathbb{F}$ such that $\widetilde{\tau}$ is a $\mathbb{G}$-stopping time and $L$ is $\mathscr{G}_{\tilde{\tau}}$-measurable. We mainly consider the forward CDS with loss in the framework of stochastic interest rates whose term structures are modeled by the Heath-Jarrow-Morton approach with jumps under the general conditional density hypothesis. We describe the dynamics of the defaultable bond in $\mathbb{G}$ and the forward CDS with random loss explicitly by the BSDEs method.
\end{abstract}

\section{Introduction}

The credit default swap (CDS) is one of the most crucial credit derivatives in the financial market and has received many attentions in recent years; see [1-5] and so forth. In the existing literature, the recovery rate of the CDS is either a constant (see [1-3], etc.) or a stochastic process. There is nothing to do with the default loss (see [4], etc.). However, the fact that once the default happens, the default loss is immediately generated and the default loss should depend on the default time is noted. From the view of the buyer of the forward CDS, he may be more interested in the credit derivative whose recovery rate depends on the default loss; that is, he hopes to obtain the higher recovery rate to avoid higher loss. More precisely, let $\widetilde{\tau}$ be the default time and let $L$ be the default loss; both $\widetilde{\tau}$ and $L$ are random variables. The recovery rate of the CDS is a function of the default loss, saying $h(L)$; that is to say, if default occurs in $[a, b]$ causing the buyer with the default loss $L$, then he can obtain $h(L)$ for the reimburse at the default time $\tilde{\tau}$. In this paper, we will consider this new kind of CDS in the framework of stochastic interest rates.
First, we assume that the filtration $\mathbb{E}$ without default is generated by a $d$-dimensional Brownian motion $W=$ $\left(W_{1}, \ldots, W_{d}\right)^{\prime}$ as well as an integer-valued random measure $\mu(d u, d y)$. In general, the default time $\tilde{\tau}$ is not an $\mathbb{F}$-stopping time; thus, the filtration of the investor is given by $\mathbb{G}=$ $\left\{\mathscr{G}_{t} ; t \geq 0\right\}$, the smallest filtration including $\mathbb{F}$ such that $\tilde{\tau}$ is a $\mathbb{G}$-stopping time and $L$ is $\mathscr{G}_{\tilde{\tau}}$-measurable.

We introduce the concept of forward CDS with random loss $L$. For any fixed $a, b$ with $0<a<b<T^{*}$, let $\mathbb{T}=\{a=$ $\left.T_{0}<T_{1}<\cdots<T_{n}=b\right\}$ be a fixed tenor structure of forward CDS; we assume that the recovery rate of the forward CDS is $h(L)$, then the discounted payoff of the protection leg for the buyer is given by

$$
\begin{aligned}
\$_{P}(t) & =\sum_{k=1}^{n} h(L) e^{-\int_{t}^{\tilde{\tau}} r(u) d u_{\coprod}}{ }_{T_{k-1}<\tau \leq T_{k}} \\
& =h(L) e^{-\int_{t}^{\tau} r(u) d u} \rrbracket_{a<\tau \leq b},
\end{aligned}
$$

where $r(u)$ is the short rate of the default-free bonds. If no default occurs before $T_{k}$, he needs to pay the fee $\mathscr{R}_{t}$ at $T_{k}$, for $k=0,1, \ldots, n-1$. It is notable that the fee $\mathscr{R}_{t}$ must be 
determined at time $t \in\left[0, T_{0}\right)$; thus, the discounted payoff of the fee leg (also known as the premium leg) equals

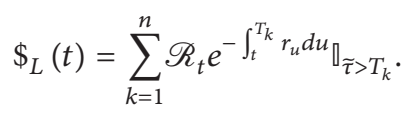

We assume that $P$ itself is an equivalent martingale measure; then the fair spread of the forward CDS for the protection buyer at $t, t \in\left[0, T_{0}\right]$, is defined as a $\mathbb{G}$-adapted process $\mathscr{R}_{t}$ such that

$$
E\left(\$_{P}(t)-\$_{L}(t) \mid \mathscr{G}_{t}\right)=0 .
$$

Since in the interval of premiums, the short rate usually changes, we assume that the short rate of the default-free bonds $r(t)$ is a $\mathbb{F}$-adapted process whose term structure is determined by the arbitrage-free HJM model with jumps (see Björk et al. [6]). In this paper, we will describe the dynamic of $\mathscr{R}_{t}$ in the frame of random interest rates by the BSDE approach.

Similar works can be found in Xiong and Kohlmann [5], in which they considered the forward CDS without default loss and their default time is modeled by the Cox model. Different from Xiong and Kohlmann [5], we assume that $(\tilde{\tau}, L)$ satisfies the following more general conditional density hypothesis (see in Jacod [7], Amendinger [8], and Callegaro et al. [9]):

Assumption 1 (the conditional density hypothesis). (i) Let $\eta(d l) d s$ be the law of $(\widetilde{\tau}, L)$ and the $\mathbb{F}$-regular conditional law of $(\tilde{\tau}, L)$ is equivalent to the law of $(\tilde{\tau}, L)$, that is,

$P\left(\tilde{\tau} \in d s, L \in d l \mid \mathscr{F}_{t}\right) \sim \eta(d l) d s, \quad$ for every $t \geq 0 ;$

(ii) $\eta(d s, d l)$ has no atoms.

Under this assumption, the immersion property is no longer valid, which leads to the problem of the enlargement of the filtration; that is, a $(P, \mathbb{F})$-martingale may be not a $(P, \mathbb{G})$ martingale, which is a fundamental problem in stochastic analysis and has been widely studied in [9-14] and so forth. By this assumption, we introduce

$$
\begin{array}{r}
W^{\mathbb{G}}(t):=W(t)+\int_{0}^{t}\left\{\left\{\frac{1}{G_{u-}}-1\right\} \widetilde{\theta}_{1}(u) \rrbracket_{u \leq \tau}\right. \\
\left.-\theta_{1}(u ; \widetilde{\tau}, L) \rrbracket_{u>\tau}\right\} d u, \\
\nu^{\mathbb{G}}(d u, d y):=\left\{1-\left\{\frac{1}{G_{u-}}-1\right\} \widetilde{\theta}_{2}(u, y) \rrbracket_{u \leq \tilde{\tau}}\right. \\
\left.+\theta_{2}(u, y ; \widetilde{\tau}, L) \mathbb{\square}_{u>\widetilde{\tau}}\right\} F_{u}(d y) d u,
\end{array}
$$

both of which depend on the default parameters $p_{s}(s, l)$, $\theta_{1}(u ; s, l) \rrbracket_{u>s}$, and $\theta_{2}(u, y ; s, l) \rrbracket_{u>s}$. One can see from Tian, Xiong, and Ye (2012), see Theorem 4.6, Corollary 4.7 and Corollary 4.8 that $W^{\mathbb{G}}$ is a $(P, \mathbb{G})$-Brownian motion and $\nu^{\mathbb{G}}(d u, d y)$ is the compensator of $\mu(d u, d y)$ with respect to
$(P, \mathbb{G})$. Further, any $(P, \mathbb{G})$-martingale $M_{t}$ can be represented into the following form

$$
\begin{aligned}
M_{t}= & M_{0}+\int_{0}^{t} \xi(u)^{\prime} d W^{\mathbb{G}}(u) \\
& +\int_{0}^{t} \int_{E} \zeta(u, y)\left\{\mu(d u, d y)-\nu^{\mathbb{G}}(d u, d y)\right\} \\
& +h(\tilde{\tau} ; \tilde{\tau}, L) \mathbb{q}_{t \geq \tilde{\tau}} \\
& -\int_{0}^{t \wedge \tau \tilde{\tau}} \int_{R} \frac{h(s ; s, l)}{G_{s-}} p_{s}(s, l) \eta(d l) d s,
\end{aligned}
$$

for some $\mathbb{G}$-predictable process $\xi, \widetilde{\mathscr{P}}(\mathbb{G})$-measurable function $\zeta(u, y)$, and a $\mathscr{O}(\mathbb{F}) \otimes \mathscr{B}\left(\mathbb{R}^{+}\right) \otimes \mathscr{B}(\mathbb{R})$-measurable function $h(u ; s, l)$.

Because of the more general conditional density hypothesis, the method of Xiong and Kohlmann [5] is no longer applicable to our case, so we extend their results and introduce a different BSDEs system depending on the given default parameters $p_{s}(s, l), \theta_{1}(u ; s, l) \rrbracket_{u>s}$, and $\theta_{2}(u, y ; s, l) \rrbracket_{u>s}$ and the term structure parameters $C(u, T)$ and $J(u, y, T)$; see (45) for more details. We show that the BSDEs system (45) always has a solution $\left(X^{d, T}, \xi_{1}^{d, T}, \xi_{2}^{d, T}\right)$, which helps us describe the predefault value of the defaultable bond explicitly. Then we introduce another BSDE depending on the recovery rate function $h(l)$ and $p_{s}(s, l)$ :

$$
\begin{aligned}
& Y_{t}= Y_{0}-\int_{0}^{t} \int_{\mathbb{R}} h(l) e^{-\int_{0}^{s} r(u) d u} \\
& \times p_{s}(s, l) \mathbb{q}_{s>a} \eta(d l) d s \\
&+ \int_{0}^{t} Y_{u-} \zeta_{1}(u)^{\prime} d W(u) \\
&+ \int_{0}^{t} Y_{u-} \zeta_{2}(u, y)\{\mu(d u, d y)-v(d u, d y)\}, \\
& Y_{b}=0,
\end{aligned}
$$

which has a solution $\left(Y, \zeta_{1}, \zeta_{2}\right)$. By the solutions of $\left(X^{d, T}, \xi_{1}^{d, T}, \xi_{2}^{d, T}\right)$ and $\left(Y, \zeta_{1}, \zeta_{2}\right)$, we describe the dynamic of the spread of the CDS more accurately.

In short, the main results are:

(1) an analysis of the conditional survival process which satisfies a special BSDE under some mild conditions; see Theorem 7;

(2) under the generally conditional density assumption, Theorems 15 and 19 describe the dynamics of the defaultable and default-free bond in $\mathbb{G}$, respectively;

(3) by Theorem 25, the dynamics of the spreads of the forward CDS is obtained, which helps us gain a deeper understanding of CDS.

The paper is organized as follows. In Section 2, we set up the stronger Jacod's hypothesis with further discussion and review martingale representation in $\mathbb{G}$. In Section 3, we 
introduce a BSDE system to describe the predefault value of the defaultable bonds, which makes us describe the dynamic of the price of the defaultable bonds more explicitly, and as a byproduct, we can also describe the dynamic of the defaultfree bonds in $\mathbb{G}$. In Section 4, we discuss the dynamics of CDS as another application of $\mathbb{G}$ where we assume that the CDS depends on the loss random variable $L$. Conclusions are given at the end.

\section{The Setup and Notations}

We assume that $\mathbb{F}=\left\{\mathscr{F}_{t} ; t \geq 0\right\}$ is the inference filtration on $(\Omega, \mathscr{F}, P)$ carrying a $d$-dimensional Brownian motion $W=$ $\left(W_{1}, \ldots, W_{d}\right)^{\prime}$ as well as an integer-valued random measure $\mu(d u, d y)$ on $\mathbb{R}_{+} \times E$, where $(E, \mathscr{E})$ is a Blackwell space. We use $\mu(d u, d y)$ to describe the change of macroeconomic policy, environment, and so forth.

Assumption 2. The filtration $\mathbb{F}=\left\{\mathscr{F}_{t} ; t \geq 0\right\}$ is the natural filtration generated by $W$ and $\mu$, that is,

$$
\begin{gathered}
\mathscr{F}_{t}=\sigma\left\{W(s), \mu\left([0, s] \times D_{1}\right), D_{2} ; 0 \leq s \leq t, D_{1} \in \mathscr{B},\right. \\
\left.D_{2} \in \mathcal{N}\right\},
\end{gathered}
$$

where $\mathcal{N}$ is the collection of $P$-null sets from $\mathscr{F}$.

In the following, we let $\mathscr{P}(\mathbb{F})$ be the family of all $\mathbb{F}$ predictable processes and $\widetilde{\mathscr{P}}(\mathbb{F})=\mathscr{P}(\mathbb{F}) \otimes \mathscr{E}$. We assume that the compensator of $\mu(d u, d y)$ is given by $v(d u, d y)=$ $F_{u}(d y) d u$, where $F_{u}(d y)$ is a transition kernel from $(\Omega \times$ $\left.\mathbb{R}_{+}, \mathscr{P}(\mathbb{F})\right)$ into $(E, \mathscr{E})$. For any local $(P, \mathbb{F})$-martingale $M$, one knows that $M$ has the representation property

$$
\begin{aligned}
M_{t}= & M_{0}+\int_{0}^{t} f_{1}(u)^{\prime} d W(u) \\
& +\int_{0}^{t} \int_{E} f_{2}(u, y)(\mu(d u, d y)-v(d u, d y)),
\end{aligned}
$$

where $f_{1}(u)$ is an $\mathbb{R}^{d}$-valued $\mathbb{F}$-predictable process with $f_{1} \in$ $L_{\text {loc }}^{2}(W)$ and $f_{2}(u, y)$ is a $\widetilde{\mathscr{P}}(\mathbb{F})$-measurable function with $f_{2} \in G_{\text {loc }}(\mu)$ (see Lemma 4.24 in page 185 of Jacod and Shiryaev [15] for more details).

Remark 3. It is easy to see that there exists an $\mathbb{F}$-optional process $\varphi=\left(\varphi_{t}\right)$ and a sequence of stopping times $\left(\widehat{\tau}_{k}\right)$ such that for all positive $\widetilde{\mathscr{P}}(\mathbb{F})$-measurable function $h(\omega, t, y)$,

$$
\int_{0}^{t} \int_{E} h(\omega, u, y) \mu(\omega ; d u, d y)=\sum_{(k)} h\left(\widehat{\tau}_{k}, \varphi_{\widehat{\tau}_{k}}\right) \mathbb{\Xi}_{\widehat{\tau}_{k} \leq t} .
$$

Furthermore, as the compensator of $\mu(d u, d y)$ is $\nu(d u, d y)=$ $F_{u}(d y) d u$, so the filtration $\mathbb{F}$ is quasi-left continuous.
Let $\tilde{\tau} \geq 0$ be the default time and let $L$ be the default loss, which are random variables. We are interested in the new enlarged filtration $\mathbb{G}=\left\{\mathscr{G}_{t} ; t \geq 0\right\}$ which is the smallest filtration including $\mathbb{F}$ such that $\tilde{\tau}$ is a $\mathbb{G}$-stopping time and $L$ is $\mathscr{G}_{\tilde{\tau}}$-measurable. We assume that $\mathbb{G}$ satisfies the usual conditions and $\mathbb{G}$ is called the progressive enlargement of $\mathbb{F}$ by $(\tilde{\tau}, L)$. We can easily see that $\mathscr{G}_{t}$ is given by

$$
\mathscr{G}_{t}=\bigcap_{u>t} \mathscr{G}_{u}^{0}, \quad \text { where } \mathscr{G}_{u}^{0}=\mathscr{F}_{u} \vee \sigma(\widetilde{\tau} \wedge u) \vee \sigma\left(L \square_{\tilde{\tau} \leq u}\right) .
$$

The information $\mathbb{G}$ means that once the default happens, the default loss is immediately generated. The similar progressive enlargement of filtration can also be found in Dellacherie and Meyer [10] and Kchia et al. [16, 17]. It is notable that the results of this progressive enlargement filtration $\mathbb{G}$ may be seen as the further extension and different from the traditional progressive enlargement of filtration in the literature; see, for example, in Jeulin [18], Jacod [7], Jeanblanc et al. [19], El Karoui et al. [11], Jeanblanc and Le Cam [12], Jeanblanc and Song [13], Callegaro et al. [9], and Jeanblanc and Song [14], among many others.

Hereinafter, we suppose that Assumptions 1 and 2 always hold. The following lemmas come from Pham [20].

Lemma 4. $Y_{t}$ is a $\mathscr{G}_{t}$-measurable random variable (r.v.) if and only if

$$
Y_{t}=y_{t}^{0} \square_{t<\tilde{\tau}}+y_{t}^{1}(\widetilde{\tau}, L) \rrbracket_{t \geq \tilde{\tau}}
$$

where $y_{t}^{0}$ is a $\mathscr{F}_{t}$-measurable r.v. and $y_{t}^{1}(s, l)$ is an $\mathscr{F}_{t} \otimes \mathscr{B}\left(\mathbb{R}^{+} \times\right.$ $\mathbb{R})$-measurable function.

Lemma 5. (1) Any $\mathbb{G}$-predictable process $Y=\left(Y_{t}\right)_{t \geq 0}$ is represented as

$$
Y_{t}=Y_{t}^{0} \rrbracket_{t \leq \tilde{\tau}}+Y_{t}^{1}(\tilde{\tau}, L) \rrbracket_{t>\tilde{\tau}},
$$

where $Y^{0}$ is a $\mathbb{F}$-predictable process and where $Y_{t}^{1}(s, l)$ is a $\mathscr{P}(\mathbb{F}) \otimes \mathscr{B}\left(\mathbb{R}^{+}\right) \otimes \mathscr{B}(\mathbb{R})$-measurable function.

(2) Any $\mathbb{G}$-optional process $Y=\left(Y_{t}\right)_{t \geq 0}$ is represented as

$$
Y_{t}=Y_{t}^{0} \rrbracket_{t<\tilde{\tau}}+Y_{t}^{1}(\tilde{\tau}, L) \rrbracket_{t \geq \tilde{\tau}},
$$

where $Y^{0}$ is an $\mathbb{F}$-optional process and where $Y_{t}^{1}(s, l)$ is $a \mathcal{O}(\mathbb{F}) \otimes$ $\mathscr{B}\left(\mathbb{R}^{+}\right) \otimes \mathscr{B}(\mathbb{R})$-measurable function.

We can see that the default time $\tilde{\tau}$ is not $\mathbb{F}$-stopping times, that is, $P(\tilde{\tau}=\tau)=0$ for every $\mathbb{F}$-stopping time $\tau$.

From Assumption 1, one can see from Jacod [7] or Amendinger [8] that there exists a strictly positive $\mathcal{O}(\mathbb{F}) \otimes$ $\mathscr{B}\left(\mathbb{R}_{+} \times \mathbb{R}\right)$-measurable function $(t ; \omega ; s, l) \rightarrow p_{t}(\omega ; s, l)$, called the $(P, \mathbb{F})$-conditional density of $(\widetilde{\tau}, L)$ with respect to $\eta$, such that for every $(s, l) \in \mathbb{R}_{+} \times \mathbb{R}, p(s, l)$ is a càdlàg $(P, \mathbb{F})$ martingale and for any $D \in \mathscr{B}\left(\mathbb{R}_{+} \times \mathbb{R}\right)$,

$$
\begin{aligned}
& P\left((\tilde{\tau}, L) \in D \mid \mathscr{F}_{t}\right)=\int_{D} p_{t}(s, l) \eta(d l) d s, \\
& \text { for every } t \geq 0, P \text {-a.s. }
\end{aligned}
$$


Since $\left\{p_{t}(s, l) ; t \geq 0\right\}$ is a strictly positive martingale, from Assumption 2 one can see that $p_{t}(s, l)$ can be represented in the following form:

$$
p_{t}(s, l)=E\left(p_{s}(s, l) \mid \mathscr{F}_{t}\right) \square_{t<s}+p_{s}(s, l) Z_{s, l}^{\theta_{1}, \theta_{2}}(t) \square_{t \geq s},
$$

where

$$
\begin{array}{r}
Z_{s, l}^{\theta_{1}, \theta_{2}}(t):=\exp \left\{\int_{s}^{t} \theta_{1}(u ; s, l)^{\prime} d W(u)-\frac{1}{2} \int_{s}^{t}\left\|\theta_{1}(u ; s, l)\right\|^{2} d u\right\} \\
\times \exp \left\{\int_{s}^{t} \int_{E}\left\{\ln \left(1+\theta_{2}(u, y ; s, l)\right)\right\} \mu(d u, d y)\right. \\
\left.-\int_{s}^{t} \int_{E} \theta_{2}(u, y ; s, l) v(d u, d y)\right\},
\end{array}
$$

and where $\theta_{1}(\cdot ; s, l)$ is an $\mathbb{R}^{d}$-valued $\mathbb{F}$-predictable process with $\theta_{1}(\cdot ; s, l) \in L_{\text {loc }}^{2}(W)$ and $\theta_{2}(\cdot, \cdot ; s, l)$ is a $\widetilde{P}(\mathbb{F})$-measurable function with $\theta_{2}(\cdot, \cdot ; s, l) \in G_{\mathrm{loc}}(\mu)$. The following theorem, from Tian et al. [21], reveals the relation of $p_{s}(s, l)$, $\theta_{1}(u ; s, l) \rrbracket_{u>s}$, and $\theta_{2}(u, y ; s, l) \rrbracket_{u>s}$.

Theorem 6. For any given bounded $p_{s}(s, l), \theta_{1}(u ; s, l) \square_{u>s}$, and $\theta_{2}(u, y ; s, l) \rrbracket_{u>s}$,

$$
p_{t}(s, l):=E\left(p_{s}(s, l) \mid \mathscr{F}_{t}\right) \rrbracket_{t<s}+p_{s}(s, l) Z_{s, l}^{\theta_{1}, \theta_{2}}(t) \rrbracket_{t \geq s}
$$

is the density process of a pair $(\widetilde{\tau}, L)$ with respect to $(P, \mathbb{F})$ if and only if

$$
\begin{gathered}
E\left(\int_{0}^{\infty} \int_{\mathbb{R}} p_{s}(s, l) \eta(d s, d l)\right)=1, \\
\int_{0}^{\infty} \int_{\mathbb{R}} p_{s}(s, l) \eta(d l) d s \\
=1-\int_{0}^{\infty}\left\{\int_{0}^{u} \int_{\mathbb{R}} p_{s}(s, l) Z_{s, l}^{\theta_{1}, \theta_{2}}(u-) \theta_{1}\right. \\
\times(u ; s, l) \eta(d l) d s\}^{\prime} d W(u) \\
-\int_{0}^{\infty} \int_{E}\left\{\int_{0}^{u} \int_{\mathbb{R}} p_{s}(s, l) Z_{s, l}^{\theta_{1}, \theta_{2}}(u-) \theta_{2}(u, y ; s, l)\right. \\
\quad \times \eta(d l) d s\}\{\mu(d u, d y)-v(d u, d y)\} .
\end{gathered}
$$

Let

$$
G_{t}:=P\left(\widetilde{\tau}>t \mid \mathscr{F}_{t}\right)=\int_{t}^{\infty} \int_{R} p_{t}(s, l) \eta(d l) d s
$$

be the conditional survival process; one can easily see that process $\left\{G_{t} ; t \geq 0\right\}$ is a $(P, \mathbb{F})$-supermartingale.
Theorem 7. For any given bounded $p_{s}(s, l), \theta_{1}(u ; s, l) \rrbracket_{u>s}$, and $\theta_{2}(u, y ; s, l) \rrbracket_{u>s}$ satisfying (19), let

$$
\begin{gathered}
\tilde{\theta}_{1}(u):=\frac{\int_{0}^{u} \int_{\mathbb{R}} p_{s}(s, l) Z_{s, l}^{\theta_{1}, \theta_{2}}(u-) \theta_{1}(u ; s, l) \eta(d l) d s}{\int_{0}^{u} \int_{\mathbb{R}} p_{s}(s, l) Z_{s, l}^{\theta_{1}, \theta_{2}}(u-) \eta(d l) d s}, \\
\widetilde{\theta}_{2}(u, y):=\frac{\int_{0}^{u} \int_{\mathbb{R}} p_{s}(s, l) Z_{s, l}^{\theta_{1}, \theta_{2}}(u-) \theta_{2}(u, y ; s, l) \eta(d l) d s}{\int_{0}^{u} \int_{\mathbb{R}} p_{s}(s, l) Z_{s, l}^{\theta_{1}, \theta_{2}}(u-) \eta(d l) d s},
\end{gathered}
$$

and then $\left(G, \widetilde{\theta}_{1}, \widetilde{\theta}_{2}\right)$ is the solution of the following BSDE:

$$
\begin{gathered}
G_{t}=1-\int_{0}^{t} \int_{\mathbb{R}} p_{s}(s, l) \eta(d l) d s \\
-\int_{0}^{t}\left\{1-G_{u-}\right\} \widetilde{\theta}_{1}(u)^{\prime} d W(u) \\
-\int_{0}^{t} \int_{E}\left\{1-G_{u-}\right\} \\
\times \widetilde{\theta}_{2}(u, y)\{\mu(d u, d y)-\nu(d u, d y)\}, \\
G_{\infty}=0 .
\end{gathered}
$$

Remark 8. It is notable that the initial value is $G_{0}=1$ and the terminal value is $G_{\infty}=0$, which is different from the traditional BSDE. In general, the BSDE (22) may not have a solution, but if $p_{s}(s, l), \theta_{1}(u ; s, l) \square_{u>s}$, and $\theta_{2}(u, y ; s, l) \rrbracket_{u>s}$ satisfy the condition (19), the BSDE (22) always has a solution.

Proof. Let

$$
I_{t}:=\int_{0}^{t} \int_{\mathbb{R}} p_{s}(s, l) Z_{s, l}^{\theta_{1}, \theta_{2}}(t) \eta(d l) d s,
$$

and then $I$ is a strictly positive $(P, \mathbb{F})$ semimartingale with $I_{t}<$ 1 , a.s. for each $t>0$. Further more,

$$
\begin{aligned}
& I_{t}=\int_{0}^{t} \int_{\mathbb{R}} p_{s}(s, l) \eta(d l) d s \\
& +\int_{0}^{t} \int_{\mathbb{R}} p_{s}(s, l) \\
& \quad \times\left\{\int_{s}^{t} Z_{s, l}^{\theta_{1}, \theta_{2}}(u-) \theta_{1}(u ; s, l)^{\prime} d W(u)\right. \\
& \quad+\int_{s}^{t} \int_{E} Z_{s, l}^{\theta_{1}, \theta_{2}}(u-) \theta_{2} \\
& \quad \times(u, y ; s, l)\{\mu(d u, d y)-\nu(d u, d y)\}\}
\end{aligned}
$$

$\times \eta(d l) d s$ 


$$
\begin{aligned}
& =\int_{0}^{t} \int_{\mathbb{R}} p_{s}(s, l) \eta(d l) d s \\
& +\int_{0}^{t}\left\{\int_{0}^{u} \int_{\mathbb{R}} p_{s}(s, l) Z_{s, l}^{\theta_{1}, \theta_{2}}(u-) \theta_{1}\right. \\
& \quad \times(u ; s, l) \eta(d l) d s\}\}^{\prime} d W(u) \\
& +\int_{0}^{t} \int_{E}\left\{\int_{0}^{u} \int_{\mathbb{R}} p_{s}(s, l) Z_{s, l}^{\theta_{1}, \theta_{2}}(u-) \theta_{2}(u, y ; s, l)\right. \\
& \quad \times \eta(d l) d s\}\{\mu(d u, d y)-v(d u, d y)\} \\
& =\int_{0}^{t} \int_{\mathbb{R}} p_{s}(s, l) \eta(d l) d s+\int_{0}^{t} I_{u-} \tilde{\theta}_{1}(u)^{\prime} d W(u) \\
& +\int_{0}^{t} \int_{E} I_{u^{-}} \tilde{\theta}_{2}(u, y)\{\mu(d u, d y)-v(d u, d y)\}
\end{aligned}
$$

and one can see that $I$ is the solution of the following SDE:

$$
\begin{aligned}
I_{t}= & \int_{0}^{t} \int_{\mathbb{R}} p_{s}(s, l) \eta(d l) d s+\int_{0}^{t} I_{u-} \widetilde{\theta}_{1}(u)^{\prime} d W(u) \\
& +\int_{0}^{t} \int_{E} I_{u-} \widetilde{\theta}_{2}(u, y)\{\mu(d u, d y)-v(d u, d y)\},
\end{aligned}
$$

and then (19) is equivalent to the following condition:

$$
I_{\infty}=1, \quad \text { a.s.; }
$$

that is to say, $\left(I, \widetilde{\theta}_{1}, \widetilde{\theta}_{2}\right)$ is a solution of the following BSDE:

$$
\begin{gathered}
I_{t}=\int_{0}^{t} \int_{\mathbb{R}} p_{s}(s, l) \eta(d l) d s+\int_{0}^{t} I_{u-} \tilde{\theta}_{1}(u)^{\prime} d W(u) \\
+\int_{0}^{t} \int_{E} I_{u-} \tilde{\theta}_{2}(u, y)\{\mu(d u, d y)-v(d u, d y)\} \\
I_{\infty}=1 .
\end{gathered}
$$

From the definition of the conditional survival process $G$, one can see that

$$
\begin{aligned}
G_{t} & =\int_{t}^{\infty} \int_{\mathbb{R}} p_{t}(s, l) \eta(d l) d s \\
& =1-\int_{0}^{t} \int_{\mathbb{R}} p_{t}(s, l) \eta(d l) d s \\
& =1-I_{t},
\end{aligned}
$$

and thus the theorem holds.

Remark 9. Let $\left(I, \widetilde{\theta}_{1}, \widetilde{\theta}_{2}\right)$ be any solution of the BSDE (27); we introduce

$$
\begin{aligned}
\theta_{1}(u ; s, l) & :=\widetilde{\theta}_{1}(u) \rrbracket_{u>s}, \\
\theta_{2}(u, y ; s, l) & :=\widetilde{\theta}_{2}(u, y) \rrbracket_{u>s},
\end{aligned}
$$

then $p_{s}(s, l), \theta_{1}(u ; s, l) \rrbracket_{u>s}$ and $\theta_{2}(u, y ; s, l) \rrbracket_{u>s}$ satisfy (19).
Example 10. If

$$
\int_{0}^{\infty} \int_{\mathbb{R}} p_{s}(s, l) \eta(d l) d s=1, \quad \text { a.s. }
$$

let $\theta_{1}(u ; s, l) \rrbracket_{u>s}:=0$ and $\theta_{2}(u, y ; s, l) \rrbracket_{u>s}:=0$; then one can see that $p_{s}(s, l), \theta_{1}(u ; s, l) \rrbracket_{u>s}$, and $\theta_{2}(u ; s, l) \rrbracket_{u>s}$ satisfy (19). Hence $\widetilde{\theta}_{1}(u)=0$ and $\widetilde{\theta}_{2}(u, y)=0$, which implies

$$
\begin{gathered}
I_{t}=\int_{0}^{t} \int_{\mathbb{R}} p_{s}(s, l) \eta(d l) d s, \\
G_{t}=1-\int_{0}^{t} \int_{\mathbb{R}} p_{s}(s, l) \eta(d l) d s .
\end{gathered}
$$

2.1. The Martingale Representation in $\mathbb{G}$. From Tian et al. [21], we have the following martingale representation theorem.

Theorem 11. Let $M_{t}:=M_{1}(t) \rrbracket_{t<\tau}+M_{2}(t ; \tilde{\tau}, L) \rrbracket_{t \geq \tau}$ be a u.i. $(P, \mathbb{G})$-martingale; then there exists a $\mathbb{G}$-predictable process $\xi$ and a $\widetilde{P}(\mathbb{G})$-measurable function $\zeta(u, y)$ such that

$$
\begin{aligned}
M_{t}= & M_{1}(0)+\int_{0}^{t} \xi(u)^{\prime} d W^{\mathbb{G}}(u) \\
& +\int_{0}^{t} \int_{E} \zeta(u, y)\left\{\mu(d u, d y)-\nu^{\mathbb{G}}(d u, d y)\right\} \\
& +M_{2}(\tilde{\tau} ; \tilde{\tau}, L) \mathbb{q}_{t \geq \tilde{\tau}}-\int_{0}^{t \wedge \tilde{\tau}} \int_{R} \frac{M_{2}(s ; s, l)}{G_{s-}} p_{s}(s, l) \eta(d l) d s \\
& -M_{1}(\tilde{\tau}-) \mathbb{q}_{t \geq \tilde{\tau}}-\int_{0}^{t \wedge \tilde{\tau}} \int_{R} \frac{M_{1}(s-)}{G_{s-}} p_{s}(s, l) \eta(d l) d s,
\end{aligned}
$$

where

$$
\begin{array}{r}
W^{\mathbb{G}}(t):=W(t)+\int_{0}^{t}\left\{\left\{\frac{1}{G_{u-}}-1\right\} \widetilde{\theta}_{1}(u) \rrbracket_{u \leq \tilde{\tau}}\right. \\
\left.\quad-\theta_{1}(u ; \tilde{\tau}, L) \rrbracket_{u>\tilde{\tau}}\right\}^{\prime} d u
\end{array}
$$

is a $(P, \mathbb{G})$-Brownian motion, and where

$$
\begin{aligned}
v^{\mathbb{G}}(d u, d y):= & \left\{1-\left\{\frac{1}{G_{u-}}-1\right\} \tilde{\theta}_{2}(u, y) \mathbb{q}_{u \leq \tilde{\tau}}\right. \\
& \left.+\theta_{2}(u, y ; \tilde{\tau}, L) \mathbb{q}_{u>\widetilde{\tau}}\right\} F_{u}(d y) d u
\end{aligned}
$$

is the compensator of $\mu(d u, d y)$ with respect to $(P, \mathbb{G})$.

Corollary 12. Let $M_{t}$ be a local $(P, \mathbb{G})$-martingale; then there exist a $\mathbb{G}$-predictable process $\xi$, a $\widetilde{P}(\mathbb{G})$-measurable function 
$\zeta(u, y)$, and a $\mathscr{O}(\mathbb{F}) \otimes \mathscr{B}\left(\mathbb{R}^{+}\right) \otimes \mathscr{B}(\mathbb{R})$-measurable function $h(u ; s, l)$ such that

$$
\begin{aligned}
M_{t}= & M_{0}+\int_{0}^{t} \xi(u)^{\prime} d W^{\mathbb{G}}(u) \\
& +\int_{0}^{t} \int_{E} \zeta(u, y)\left\{\mu(d u, d y)-\nu^{\mathbb{G}}(d u, d y)\right\} \\
& +h(\tilde{\tau} ; \tilde{\tau}, L) \mathbb{\square}_{t \geq \tilde{\tau}}-\int_{0}^{t \wedge \tilde{\tau}} \int_{R} \frac{h(s ; s, l)}{G_{s^{-}}} p_{s}(s, l) \eta(d l) d s .
\end{aligned}
$$

\section{The Dynamics of the Default-Free Bond in $\mathbb{G}$}

We now discuss the market of the defaultable-free bonds. Since $\mathbb{F}$ is the filtration without default, it is natural to assume that the price processes of the default-free bonds are $\mathbb{F}$ semimartingale. We assume that $P$ itself is an equivalent martingale measure and the short rate is modeled by the arbitrage-free HJM model with jumps (see Björk et al. [6]). More precisely, let $B^{\mathbb{F}}(t, T)$ be the price at time $t$ of the defaultfree bond maturing at time $T$ (a $T$-bond), then $\left\{B^{\mathbb{F}}(t, T) ; t \in\right.$ $[0, T]\}$ is an $\mathbb{F}$ semimartingale with $B^{\mathbb{F}}(T, T)=1$ for each $T>0$. To discuss the term structure of the default-free bond, we assume that, for every fixed $t, B^{\mathbb{F}}(t, T)$ is $P$-a.s. continuously differentiable in the $T$-variable, and introduce the instantaneous forward rate $f(t, T)=-(\partial / \partial T) \log B^{\mathbb{F}}(t, T)$ and the short rate $r(t)=f(t, t)$, as in [6]. If the object $\xi$ is continuously differentiable in the $T$-variable, then we denote the partial $T$-derivative by $\xi_{T}$.

For the simplicity, we assume that $P$ is an equivalent martingale measure for $B^{\mathbb{F}}(t, T)$ for any maturity $T$; that is, the discount process $\left\{e^{-\int_{0}^{t} r(u) d u} B^{\mathbb{F}}(t, T) ; t \in[0, T]\right\}$ is a $\mathbb{F}$ martingale, and the dynamics of $f(t, T)$ is given by

$$
\begin{aligned}
f(t, T)= & f(0, T)+\int_{0}^{t} \alpha(u, T) d u+\int_{0}^{t} \beta(u, T)^{\prime} d W(u) \\
& +\int_{0}^{t} \int_{E} \gamma(u, y, T) \mu(d u, d y)
\end{aligned}
$$

where $\alpha(\cdot, T)$ is a bounded $\mathbb{F}$-predictable process on $[0, T]$, $\beta(\cdot, T)$ is a bounded $\mathbb{R}^{d}$-valued $\mathbb{F}$-predictable process on $[0, T]$ and where $\gamma(\cdot, \cdot, T)$ is a bounded $\widetilde{\mathscr{P}}(\mathbb{F})$-measurable function on $[0, T] \times E$ for each $T$. Since it is arbitrage free, one can see from [6] or [22] that

$$
\alpha(u, T)=-\beta(u, T)^{\prime} C(u, T)-\int_{E} \gamma(u, y, T) e^{J(u, y, T)} F_{u}(d y),
$$

where

$$
\begin{gathered}
C(u, T):=-\int_{u}^{T} \beta(u, l) d l, \\
J(u, y, T):=-\int_{u}^{T} \gamma(u, y ; l) d l .
\end{gathered}
$$

The following lemma can be found in Proposition 2.4 of [6] or Lemma 2.3 of [22].

Lemma 13. If the dynamics of the forward rate $f(t, T)$ is given by (36), then the short rate $r(t)$ satisfies

$$
\begin{aligned}
r(t)= & r(0)+\int_{0}^{t} \alpha^{*}(u) d u+\int_{0}^{t} \beta(u, u)^{\prime} d W(u) \\
& +\int_{0}^{t} \int_{E} \gamma(u, y, u) \mu(d u, d y),
\end{aligned}
$$

where $r(0)=f(0,0), \alpha^{*}(u)=f_{T}(u, u)+\alpha(u, u)$, and the dynamics $B^{\mathbb{F}}(t, T)$ is given by

$$
\begin{aligned}
B^{\mathbb{F}}(t, T)= & B^{\mathbb{F}}(0, T)+\int_{0}^{t} B^{\mathbb{F}}(u-, T) r(u) d u \\
& +\int_{0}^{t} B^{\mathbb{F}}(u-, T) C(u, T)^{\prime} d W(u) \\
& +\int_{0}^{t} \int_{E} B^{\mathbb{F}}(u-, T)\left\{e^{J(u, y, T)}-1\right\} \\
& \times\{\mu(d u, d y)-v(d u, d y)\} .
\end{aligned}
$$

Remark 14. From (40), one can see that

$$
\begin{aligned}
& -\int_{t}^{T} f(t, l) d l \\
& =-\int_{0}^{T} f(0, l) d l \\
& \quad+\int_{0}^{t}\left\{r(u)-\left\{\frac{1}{2}\|C(u, T)\|^{2}\right.\right. \\
& \quad+\int_{0}^{t} C(u, T)^{\prime} d W(u) \\
& \left.\left.\left.\quad+\int_{0}^{t} \int_{E}^{J(u, y, T)}-1\right\} F_{u}(d y)\right\}\right\} d u
\end{aligned}
$$

If we take $t=T$, one gets

$$
\begin{aligned}
\int_{0}^{T} r(u) d u= & \int_{0}^{T} f(0, l) d l \\
& +\int_{0}^{T}\left\{\frac{1}{2}\|C(u, T)\|^{2}\right. \\
& \left.\quad+\int_{E}\left\{e^{J(u, y, T)}-1\right\} F_{u}(d y)\right\} d u \\
& -\int_{0}^{T} C(u, T)^{\prime} d W(u) \\
& -\int_{0}^{T} \int_{E} J(u, y, T) \mu(d u, d y) .
\end{aligned}
$$


3.1. The Price Process of the Defaultable Bond. Let $B^{d}(t, T)$ be the time- $t$ price of the defaultable zero coupon bond with zero recovery and with maturity $T$, that is,

$$
\begin{aligned}
& B^{d}(t, T)=E\left(e^{-\int_{t}^{T} r(u) d u_{\rrbracket_{\tau}>T}} \mid \mathscr{G}_{t}\right) \\
& =\frac{1}{G_{t}} E\left(\int_{T}^{\infty} \int_{\mathbb{R}} e^{-\int_{t}^{T} r(u) d u} p_{T}(s, l) \eta(d l) d s \mid \mathscr{F}_{t}\right) \rrbracket_{t<\tau} \\
& =E\left(e^{-\int_{t}^{T} r(u) d u} \frac{G_{T}}{G_{t}} \mid \mathscr{F}_{t}\right) \rrbracket_{t<\tau} \\
& =B^{*}(t, T) \mathbb{\square}_{t<\tau} \text {, }
\end{aligned}
$$

where $B^{*}(t, T)=E\left(e^{-\int_{t}^{T} r(u) d u}\left(G_{T} / G_{t}\right) \mid \mathscr{F}_{t}\right)$ is the predefault value of the default bond $B^{d}(t, T)$.

We first review the following case which is usually called the Cox model. Given a nonnegative bounded $\mathbb{F}$-predictable process $\lambda=\{\lambda(u) ; u \geq 0\}$ with $\int_{0}^{\infty} \lambda(u) d u=\infty$, a.s. Let $\Theta$ be a random variable (r.v.) independent to $\mathscr{F}_{\infty}$ with unit exponential law; then the default time is defined as

$$
\tilde{\tau}=\inf \left\{t: \int_{0}^{t} \lambda(u) d u \geq \Theta\right\}
$$

see Lando [23]. For Cox model, $\lambda(u)$ is the so-called intensity process and $B^{*}(t, T)=E\left(e^{-\int_{t}^{T}(r(u)+\lambda(u)) d u} \mid \mathscr{F}_{t}\right)$; also see Lando [23]. However, under the conditional density assumption, $B^{*}(t, T)$ is still not clear and we want to describe the dynamic of $B^{*}(t, T)$, so we introduce the following BSDEs system:

$$
\begin{aligned}
& X_{t}^{d, T}=X_{0}^{d, T}+\int_{0}^{t}\left\{\xi_{1}^{d, T}(u)-C(u, T)\right. \\
& \left.+\left\{\frac{1}{G_{u-}}-1\right\} \widetilde{\theta}_{1}(u)\right\}^{\prime} d W(u) \\
& +\frac{1}{2} \int_{0}^{t}\left\{\left\{\frac{1}{G_{u-}}-1\right\}^{2}\left\|\widetilde{\theta}_{1}(u)\right\|^{2}\right. \\
& \left.-\left\|\xi_{1}^{d, T}(u)\right\|^{2}+\|C(u, T)\|^{2}\right\} d u \\
& +\int_{0}^{t} \int_{E}\left\{\ln \left(1+\xi_{2}^{d, T}(u, y)\right)\right. \\
& -\ln \left(1+\left(\frac{1}{G_{u-}}-1\right) \tilde{\theta}_{2}(u, y)\right) \\
& -J(u, y ; T)\} \mu(d u, d y)
\end{aligned}
$$

$$
\begin{gathered}
+\int_{0}^{T} \int_{E}\left\{\left\{e^{J(u, y, T)}-1\right\}-\xi_{2}^{d, T}(u, y)\right. \\
\left.-\left\{\frac{1}{G_{u-}}-1\right\} \tilde{\theta}_{2}(u, y)\right\} F_{u}(d y) \\
+\int_{0}^{t} \int_{\mathbb{R}} \frac{1}{G_{s-}} p_{s}(s, l) \eta(d l) d s, \\
X_{T}^{d, T}=0 .
\end{gathered}
$$

The solution of the system of BSDEs (45) is a triplet $\left(X^{d, T}, \xi_{1}^{d, T}, \xi_{2}^{d, T}\right)$ satisfying (45) such that for any $T \in$ $\left[0, T^{*}\right],\left\{\xi_{1}^{d, T}(u ; T) ; u \in[0, T]\right\}$ is an $\mathbb{R}^{n}$-valued $\mathbb{F}$-predictable process and $\xi_{2}^{d, T}(u, y ; T)$ is a $\widetilde{P}(\mathbb{F})$-measurable function such that the stochastic exponential $\mathscr{E}\left\{\int_{0}^{\cdot} \xi_{1}^{d, T}(u)^{\prime} d W(u)+\right.$ $\left.\int_{0}^{\cdot} \int_{E} \xi_{2}^{d, T}(u, y)\{\mu(d u, d y)-v(d u, d y)\}\right\}_{t}$ is a uniformly integrable $(P, \mathbb{F})$-martingale on $[0, T]$.

Theorem 15. (i) The system of BSDEs (45) has a solution.

(ii) Let $\left(X^{d, T}, \xi_{1}^{d, T}, \xi_{2}^{d, T}\right)$ be any solution of the system of BSDEs (45); then for any maturity $T \in\left[0, T^{*}\right]$ and for any $t \leq T, B^{*}(t, T)$ satisfies the following SDE

$B^{*}(t, T)$

$$
\begin{aligned}
& =e^{X_{0}^{d, T}-\int_{0}^{T} f(0, l) d l} \\
& +\int_{0}^{t} \int_{\mathbb{R}} B^{*}(s-, T) \frac{1}{G_{s}} p_{s}(s, l) \eta(d l) d s \\
& +\int_{0}^{t} B^{*}(u-, T) \\
& \times\left\{r(u)+\left\|\left\{\frac{1}{G_{u-}}-1\right\} \widetilde{\theta}_{1}(u)+\frac{1}{2} \xi_{1}^{d, T}(u)\right\|^{2}\right. \\
& \left.-\frac{1}{4}\left\|\xi_{1}^{d, T}(u)\right\|^{2}\right\} d u \\
& +\int_{0}^{t} \int_{E} B^{*}(u-, T)\left\{\frac{1}{G_{u-}}-1\right\} \widetilde{\theta}_{2}(u, y) \\
& \times\left\{\frac{1+\xi_{2}^{d, T}(u, y)}{1-\left\{1 / G_{u-}-1\right\} \widetilde{\theta}_{2}(u, y)}-1\right\} F_{u}(d y) d u \\
& +\int_{0}^{t} B^{*}(u-, T)\left\{\left\{\frac{1}{G_{u-}}-1\right\} \widetilde{\theta}_{1}(u)\right. \\
& \left.+\xi_{1}^{d, T}(u)\right\}^{\prime} d W(u) \\
& +\int_{0}^{t} \int_{E} B^{*}(u-, T)
\end{aligned}
$$




$$
\begin{aligned}
& \times\left\{\frac{1+\xi_{2}^{d, T}(u, y)}{1-\left\{1 / G_{u-}-1\right\} \widetilde{\theta}_{2}(u, y)}\right. \\
& -1\}\{\mu(d u, d y)-v(d u, d y)\} .
\end{aligned}
$$

The proof is given in Appendix.

Remark 16. From (46), one can see that $B^{*}(t, T)$ can be rewritten as the follows:

$$
B^{*}(t, T)=E\left(e^{-\int_{t}^{T}\{r(u)+\delta(u, T)\} d u} \mid \mathscr{F}_{t}\right),
$$

where

$$
\begin{aligned}
\delta(u, T):= & \frac{1}{2}\left\|\left\{\frac{1}{G_{u-}}-1\right\} \widetilde{\theta}_{1}(u)+\xi_{1}^{d, T}(u)\right\|^{2} \\
& -\frac{1}{2}\left\|\xi_{1}^{d, T}(u)\right\|^{2}+\int_{\mathbb{R}} \frac{1}{G_{u}} p_{u}(u, l) \eta(d l) \\
& +\int_{E}\left\{\frac{1}{G_{u-}}-1\right\} \widetilde{\theta}_{2}(u, y) \\
& \times\left\{\frac{1+\xi_{2}^{d, T}(u, y)}{1-\left\{1 / G_{u-}-1\right\} \widetilde{\theta}_{2}(u, y)}-1\right\} F_{u}(d y)
\end{aligned}
$$

is the spread of the defaultable bond with respect to the default-free bond $B^{\mathbb{F}}(t, T)$, which may be negative.

Remark 17. If

$$
\int_{0}^{\infty} \int_{\mathbb{R}} p_{s}(s, l) \eta(d l) d s=1, \quad \text { a.s., }
$$

as in Example 10, then the BSDEs system (45) can be simplified into the following form:

$$
\begin{aligned}
& X_{t}^{d, T}= X_{0}^{d, T}+\int_{0}^{t}\left\{\xi_{1}^{d, T}(u)-C(u, T)\right\}^{\prime} d W(u) \\
&-\frac{1}{2} \int_{0}^{t}\left\{\left\|\xi_{1}^{d, T}(u)\right\|^{2}-\|C(u, T)\|^{2}\right\} d u \\
&+\int_{0}^{t} \int_{E}\left\{\ln \left(1+\xi_{2}^{d, T}(u, y)\right)-J(u, y ; T)\right\} \mu(d u, d y) \\
&+\int_{0}^{t} \int_{E}\left\{\left\{e^{J(u, y, T)}-1\right\}-\xi_{2}^{d, T}(u, y)\right\} F_{u}(d y) \\
&+\int_{0}^{t} \int_{\mathbb{R}} \frac{1}{G_{s-}} p_{s}(s, l) \eta(d l) d s, \\
& X_{T}^{d, T}=0,
\end{aligned}
$$

which can be transformed into the BSDEs system (1.3) of Xiong and Kohlmann [5], so the BSDEs system (45) may be viewed as an extension of [5].
Corollary 18. Let $\left(X^{d, T}, \xi_{1}^{d, T}, \xi_{2}^{d, T}\right)$ be any solution of the system of BSDEs (45), then for any maturity $T \in\left[0, T^{*}\right]$ and for any $t \leq T, B^{d}(t, T)$ satisfies the following SDE:

$$
\begin{aligned}
& B^{d}(t, T)=e^{X_{0}^{d, T}-\int_{0}^{T} f(0, l) d l} \\
& +\int_{0}^{t \wedge \tilde{\tau}} B^{d}(u-, T) r(u) d u \\
& +\int_{0}^{t \wedge \tilde{\tau}} B^{d}(u-, T)\left\{\left\{\frac{1}{G_{u-}}-1\right\} \tilde{\theta}_{1}(u)\right. \\
& \left.+\xi_{1}^{d, T}(u)\right\}^{\prime} d W^{\mathbb{G}}(u) \\
& +\int_{0}^{t \wedge \tilde{\tau}} \int_{E} B^{d}(u-, T) \\
& \times\left\{\frac{1+\xi_{2}^{d, T}(u, y)}{1-\left\{1 / G_{u^{-}}-1\right\} \widetilde{\theta}_{2}(u, y)}\right. \\
& -1\}\left\{\mu(d u, d y)-v^{\mathbb{G}}(d u, d y)\right\} \\
& -B^{d}(\tilde{\tau}-, T) \rrbracket_{t \geq \tilde{\tau}} \\
& +\int_{0}^{t \wedge \tilde{\tau}} \int_{\mathbb{R}} B^{d}(s-, T) \frac{1}{G_{s}} p_{s}(s, l) \eta(d l) d s .
\end{aligned}
$$

Proof. Since $B^{d}(t, T)=B^{*}(t, T) \rrbracket_{t<\tau}$, one can see that $B^{d}(\tilde{\tau}-, T)=B^{*}(\tilde{\tau}, T)$ and $B^{d}(u-, T) \rrbracket_{u \leq \tau}=B^{*}(u-, T) \rrbracket_{u \leq \tilde{\tau}}$; then $\operatorname{SDE}(51)$ follows from $B^{d}(t, T)=B^{*}(t \wedge \tilde{\tau}, T)-$ $B^{*}(\tilde{\tau}, T) \rrbracket_{t \geq \tilde{\tau}}$.

3.2. The Price Process of the Default-Free Bond in $\mathbb{G}$. It is easy to see that

$$
B^{\mathbb{F}}(t, T)=E\left(e^{-\int_{t}^{T} r(u) d u} \mid \mathscr{F}_{t}\right)
$$

and from (40), one can see that $B^{\mathbb{F}}$ is completely determined by $A, C$, and $J$. Although $\mathbb{F}$ is the filtration without default, the filtration $\mathbb{G}$ can be observed by all investor; thus, it is also interesting to consider the dynamics of $B(t, T)$, where

$$
B(t, T):=E\left(e^{-\int_{t}^{T} r(u) d u} \mid \mathscr{G}_{t}\right) .
$$


We need to introduce the following BSDEs system:

$$
\begin{aligned}
& X_{t}^{T}=X_{0}^{T}+\int_{0}^{t}\left\{\xi_{1}^{T}(u)-C(u, T)\right\}^{\prime} d W^{\mathbb{G}}(u) \\
& +\int_{0}^{t}\left\{-\frac{1}{2}\left\|\xi_{1}^{T}(u)\right\|^{2}+\frac{1}{2}\|C(u, T)\|^{2}\right. \\
& +C(u, T)^{\prime}\left\{\left\{\frac{1}{G_{u-}}-1\right\} \tilde{\theta}_{1}(u) \mathbb{q}_{u \leq \tilde{\tau}}\right. \\
& \left.\left.-\theta_{1}(u ; \tilde{\tau}, L) \mathbb{q}_{u>\tau}\right\}\right\} d u \\
& +\int_{0}^{t} \int_{E}\left\{\ln \left\{1+\xi_{2}^{T}(u, y)\right\}-J(u, y ; T)\right\} \mu(d u, d y) \\
& +\int_{0}^{t} \int_{E}\left\{\left\{e^{J(u, y, T)}-1\right\}-\xi_{2}^{T}(u, y)\right. \\
& \times\left\{1-\left\{\frac{1}{G_{u-}}-1\right\} \tilde{\theta}_{2}(u, y) \mathbb{q}_{u \leq \tilde{\tau}}\right. \\
& \left.\left.+\theta_{2}(u, y ; \widetilde{\tau}, L) \mathbb{q}_{u>\widetilde{\tau}}\right\}\right\} F_{u}(d y) d u \\
& +\ln \left\{1+h^{T}(\tilde{\tau} ; \tilde{\tau}, L)\right\} \rrbracket_{t \geq \tilde{\tau}} \\
& -\int_{0}^{t \wedge \tau} \int_{R} h^{T}(s ; s, l) \frac{1}{G_{s-}} p_{s}(s, l) \eta(d l) d s, \\
& X_{T}=0 \text {. }
\end{aligned}
$$

The solution of the BSDEs system (54) is $\left(X^{T}, \xi_{1}^{T}, \xi_{2}^{T}, h^{T}\right)$ such that it satisfies (54) such that for any $T \in\left[0, T^{*}\right]$, $\left\{\xi_{1}^{T}(u) ; u \in[0, T]\right\}$ is an $\mathbb{R}^{n}$-valued $\mathbb{G}$-predictable process, $\xi_{2}^{T}(u, y)$ is a $\widetilde{P}(\mathbb{G})$-measurable function, and $h^{T}(s ; s, l)$ is a $\mathscr{O}(\mathbb{F}) \otimes \mathscr{B}\left(\mathbb{R}^{+}\right) \otimes \mathscr{B}(\mathbb{R})$-measurable function such that that the stochastic exponential $\mathscr{E}\left\{\int_{0}^{\cdot} \xi_{1}^{d, T}(u)^{\prime} d W^{\mathbb{G}}(u)+\right.$ $\int_{0}^{\cdot} \int_{E} \xi_{2}^{d, T}(u, y)\left\{\mu(d u, d y)-v^{\mathbb{G}}(d u, d y)\right\}+h^{T}(\tilde{\tau} ; \tilde{\tau}, L) \rrbracket_{\cdot \geq \tilde{\tau}}-$ $\left.\int_{0}^{\cdot \wedge \tilde{\tau}} \int_{R} h^{T}(s ; s, l)\left(1 / G_{s-}\right) p_{s}(s, l) \eta(d l) d s\right\}_{t}$ is a uniformly integrable $(P, \mathbb{G})$-martingale on $[0, T]$.

Theorem 19. (i) The BSDEs system (54) has a solution.

(ii) Let $\left(X^{T}, \xi_{1}^{T}, \xi_{2}^{T}, h^{T}\right)$ be any solution of the system of $B S D E s$ (54); then $B(t, T)$ satisfies the following SDE:

$$
\begin{aligned}
B(t, T)= & e^{X_{0}^{T}-\int_{0}^{T} f(0, l) d l} \\
& +\int_{0}^{t} B(u-, T) r(u) d u \\
& +\int_{0}^{t} B(u-, T) \xi_{1}^{T}(u)^{\prime} d W^{\mathbb{G}}(u)
\end{aligned}
$$

$$
\begin{aligned}
& +\int_{0}^{t} \int_{E} B(u-, T) \xi_{2}^{T}(u, y) \\
& \quad \times\left\{\mu(d u, d y)-\nu^{\mathbb{G}}(d u, d y)\right\} \\
& +B(\tilde{\tau}-, T) h^{T}(\tilde{\tau} ; \tilde{\tau}, L) \mathbb{\square}_{t \geq \tilde{\tau}} \\
& -\int_{0}^{t \wedge \tilde{\tau}} \int_{R} B(s-, T) h^{T}(s ; s, l) \frac{1}{G_{s-}} p_{s}(s, l) \eta(d l) d s .
\end{aligned}
$$

The proof is given in Appendix.

Remark 20. Since the BSDEs system (54) not only depends on $A, C, J$, but also depends on $\theta_{1}(u ; s, l), \theta_{2}(u, y ; s, l), \widetilde{\theta}_{1}(u)$, and $\widetilde{\theta}_{2}(u, y)$, the dynamics of the price process of the defaultfree bond in $\mathbb{G}$ is affected by both the default time and the loss.

Remark 21. Although $r$ is a $\mathbb{F}$ semimartingale, which is not affected by the default, there still exists a jump in $B(t, T)$ at the default time.

\section{The Dynamics of the Forward CDS}

In this section, we will describe the dynamics of the spreads of the forward CDS. For any fixed $a<b$ with $0<a<b<T^{*}$, let $\pi=\left\{a=T_{0}<T_{1}<\cdots<T_{n}=b\right\}$ be a fixed tenor structure. We assume that the recovery rate is determined by a process $h(L)$ with $0<h(L)<1$, for $u \in\left[0, T^{*}\right]$; that is, if default occurs between the dates $T_{k-1}$ and $T_{k}$, the buyer of the CDS will receive the protection payment $h(L)$ at time $\tilde{\tau}$. The random variable $h(L)$ depends on the loss $L$ when the default occurs, which is different from $[3,5]$. For example, $h(L)=\delta_{1} I_{L<a}+\delta_{2} I_{L \geq a}$; that is to say, if $L<a$, the recovery rate is $\delta_{1}$, and if $L>a$, the recovery rate is $\delta_{2},\left(\delta_{2}>\delta_{1}\right)$.

We assume that $t<T_{0}$ and the forward credit default swap (forward CDS) issued at time $t \in\left[0, T_{0}\right]$, with the unit notional. The discounted payoff of the protection leg equals

$$
\begin{aligned}
\$_{P}(t) & =\sum_{k=1}^{n} h(L) e^{-\int_{t}^{\tilde{\tau}} r(u) d u} \rrbracket_{T_{k-1}<\tilde{\tau} \leq T_{k}} \\
& =h(L) e^{-\int_{t}^{\tilde{\tau}} r(u) d u} \rrbracket_{a<\tilde{\tau} \leq b}
\end{aligned}
$$

and the discounted payoff of the fee leg (also known as the premium leg) equals

$$
\$_{L}(t)=\sum_{k=1}^{n} e^{-\int_{t}^{T_{k}} r(u) d u} \rrbracket_{\widetilde{\tau}>T_{k}} .
$$

The following definition is adapted from [3].

Definition 22. The fair spread of the forward CDS with enlarged filtration $\mathbb{G}$ for the protection buyer equals, for 
every $t \in\left[0, T_{0}\right]$, is defined as a $\mathscr{G}_{t}$-adapted process $\mathscr{R}_{t}$ such that $E\left(\$_{P}(t)-\mathscr{R}_{t} \$_{L}(t) \mid \mathscr{G}_{t}\right)=0$; that is,

$$
\mathscr{R}_{t}=\frac{E\left(\$_{P}(t) \mid \mathscr{G}_{t}\right)}{E\left(\$_{L}(t) \mid \mathscr{G}_{t}\right)} .
$$

We have the following lemma.

Lemma 23. Let $B^{*}(t, T)$ be the predefault value of the defaultable bond, and let

$$
\begin{aligned}
& D(t, a, b) \\
& :=\frac{1}{G_{t}} e^{\int_{0}^{t} r(u) d u} \\
& \quad \times E\left(\int_{a}^{b} \int_{\mathbb{R}} h(l) e^{-\int_{0}^{s} r(u) d u} p_{s}(s, l) \eta(d l) d s \mid \mathscr{F}_{t}\right),
\end{aligned}
$$

and then we have, for $t \leq a$,

$$
\mathscr{R}_{t}=\frac{D(t, a, b)}{\sum_{k=1}^{n} B^{*}\left(t, T_{k}\right)} \rrbracket_{t<\tau} .
$$

Proof. Since, for $t \leq a$,

$$
\begin{aligned}
& E\left(\$_{P}(t) \mid \mathscr{G}_{t}\right)
\end{aligned}
$$

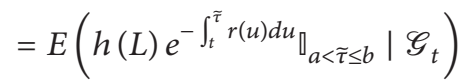

$$
\begin{aligned}
& =\frac{1}{G_{t}} E\left(\int_{a}^{b} \int_{\mathbb{R}} h(l) e^{-\int_{t}^{s} r(u) d u} p_{b}(s, l) \eta(d l) d s \mid \mathscr{F}_{t}\right) \mathbb{q}_{t<\tau} \\
& =\frac{1}{G_{t}} e^{\int_{0}^{t} r(u) d u} \\
& \times E\left(\int_{a}^{b} \int_{\mathbb{R}} h(l) e^{-\int_{0}^{s} r(u) d u} p_{s}(s, l) \eta(d l) d s \mid \mathscr{F}_{t}\right) \rrbracket_{t<\tau} \\
& =D(t, a, b) \rrbracket_{t<\tilde{\tau}}, \\
& E\left(\$_{L}(t) \mid \mathscr{G}_{t}\right) \\
& =E\left(\sum_{k=1}^{n} e^{-\int_{t}^{T_{k}} r(u) d u_{\rrbracket_{\tilde{\tau}>T_{k}}}} \mathscr{G}_{t}\right) \\
& =\frac{1}{G_{t}} \sum_{k=1}^{n} e^{\int_{0}^{t} r(u) d u} \\
& \times E\left(e^{-\int_{0}^{T_{k}} r(u) d u} \int_{T_{k}}^{\infty} \int_{\mathbb{R}} p_{T_{k}}(s, l) \eta(d l) d s \mid \mathscr{F}_{t}\right) \rrbracket_{t<\tau} \\
& =\frac{1}{G_{t}} e^{\int_{0}^{t} r(u) d u} \sum_{k=1}^{n} E\left(e^{-\int_{0}^{T_{k}} r(u) d u} G_{T_{k}} \mid \mathscr{F}_{t}\right) \mathbb{\varpi}_{t<\tilde{\tau}} \\
& =\sum_{k=1}^{n} B^{*}\left(t, T_{k}\right) \mathbb{q}_{t<\tilde{\tau}}
\end{aligned}
$$

(60) follows from the definition of $\mathscr{R}_{t}$.
To describe the dynamic of the spread, we need to introduce the BSDE (7) depending on $h(l)$.

The solution of the BSDE $(7)$ is $\left(Y, \zeta_{1}, \zeta_{2}\right)$ where $\left\{\zeta_{1}(u) ; u \in\right.$ $[a, b]\}$ is an $\mathbb{R}^{n}$-valued $\mathbb{F}$-predictable process and $\zeta_{2}(u, y)$ is a $\widetilde{\mathscr{P}}(\mathbb{F})$-measurable function, such that the stochastic exponential $\mathscr{E}\left\{\int_{0}^{\cdot} \zeta_{1}(u)^{\prime} d W(u)+\int_{0}^{\cdot} \int_{E} \zeta_{2}(u, y)\{\mu(d u, d y)-\right.$ $v(d u, d y)\}\}_{t}$ is a uniformly integrable $(P, \mathbb{F})$-martingale on $[a, b]$.

One can see that the BSDE (7) always has a solution, such that

$$
Y_{t}=E\left[\int_{t}^{b} \int_{\mathbb{R}} h(l) e^{-\int_{0}^{s} r(u) d u} p_{s}(s, l) \mathbb{q}_{s>a} \eta(d l) d s \mid \mathscr{F}_{t}\right] .
$$

Remark 24. Let $\left(Y^{a, b}, \zeta_{1}^{a, b}, \zeta_{2}^{a, b}\right)$ be the solution of the BSDE (7), then

$$
\begin{aligned}
Y_{t}+ & \int_{0}^{t} \int_{\mathbb{R}} h(l) e^{-\int_{0}^{s} r(u) d u} p_{s}(s, l) \rrbracket_{s>a} \eta(d l) d s \\
= & E\left[\int_{a}^{b} \int_{\mathbb{R}} h(l) e^{-\int_{0}^{s} r(u) d u} p_{s}(s, l) \eta(d l) d s \mid \mathscr{F}_{t}\right] \\
= & Y_{0}+\int_{0}^{t} Y_{u-} \zeta_{1}(u)^{\prime} d W(u) \\
& +\int_{0}^{t} Y_{u-} \zeta_{2}(u, y)\{\mu(d u, d y)-v(d u, d y)\},
\end{aligned}
$$

which implies that the restricted process $\left\{Y_{t}, t \in[0, a]\right\}$ is a $(P, \mathbb{F})$-martingale; that is to say, for $t<a, Y$ satisfies the following SDE:

$$
\begin{aligned}
Y_{t}= & Y_{0}+\int_{0}^{t} Y_{u-} \zeta_{1}(u)^{\prime} d W(u) \\
& +\int_{0}^{t} Y_{u-} \zeta_{2}(u, y)\{\mu(d u, d y)-v(d u, d y)\} .
\end{aligned}
$$

Theorem 25. Assume that $\left(Y, \zeta_{1}, \zeta_{2}\right)$ is the solution of the BSDE (7) and $\left(X^{d, T_{k}}, \xi_{1}^{d, T_{k}}, \xi_{2}^{d, T_{k}}\right)$ is the solution of the system of BSDEs (45); let

$$
\begin{aligned}
M_{t}^{k}= & \mathscr{E} \\
& \left(\int_{0}^{\cdot} \xi_{1}^{d, T_{k}}(u)^{\prime} d W(u)\right. \\
& \left.+\int_{0}^{\cdot} \int_{E} \xi_{2}^{d, T_{k}}(u, y)\{\mu(d u, d y)-v(d u, d y)\}\right)_{t},
\end{aligned}
$$


and assume that $\mathscr{R}_{t}^{*}$ is the predefault vaule of the CDS, that is, $\mathscr{R}_{t}=\mathscr{R}_{t}^{*} \square_{t<\tau}$, and then for $t<a, \mathscr{R}_{t}^{*}$ satisfies the following $S D E$

$$
\begin{aligned}
& \mathscr{R}_{t}^{*}=\frac{Y_{0}}{M_{0}} \\
& -\int_{0}^{t} \mathscr{R}_{u-}^{*}\left\{\frac{\sum_{k=1}^{n} M_{u-}^{k} \xi_{1}^{d, T_{k}}(u)}{\sum_{k=1}^{n} M_{u-}^{k}}-\zeta_{1}(u)\right\}^{\prime} d W(u) \\
& +\int_{0}^{t} \int_{E} \mathscr{R}_{u-}^{*}\left\{\frac{1+\zeta_{2}(u, y)}{1+\sum_{k=1}^{n} M_{u^{-}}^{k} \xi_{2}^{d, T_{k}}(u, y) / \sum_{k=1}^{n} M_{u-}^{k}}\right. \\
& -1\}\{\mu(d u, d y)-v(d u, d y)\} \\
& +\int_{0}^{t} \mathscr{R}_{u-}^{*} \frac{\sum_{k=1}^{n} M_{u-}^{k} \xi_{1}^{d, T_{k}}(u)^{\prime}}{\sum_{k=1}^{n} M_{u-}^{k}} \\
& \times\left\{\frac{\sum_{k=1}^{n} M_{u-}^{k} \xi_{1}^{d, T_{k}}(u)}{\sum_{k=1}^{n} M_{u-}^{k}}-\zeta_{1}(u)\right\} d u \\
& +\int_{0}^{t} \int_{E} \mathscr{R}_{u-}^{*}\left\{\frac{\sum_{k=1}^{n} M_{u-}^{k} \xi_{2}^{d, T_{k}}(u, y)}{\sum_{k=1}^{n} M_{u-}^{k}}-\zeta_{2}(u, y)\right\} \\
& \times \frac{\sum_{k=1}^{n} M_{u-}^{k} \xi_{2}^{d, T_{k}}(u, y) / \sum_{k=1}^{n} M_{u-}^{k}}{1+\sum_{k=1}^{n} M_{u-}^{k} \xi_{2}^{d, T_{k}}(u, y) / \sum_{k=1}^{n} M_{u-}^{k}} F_{u}(d y) d u,
\end{aligned}
$$

where $M_{0}:=\sum_{k=1}^{n} e^{X_{0}^{d, T_{k}}-\int_{0}^{T_{k}} f(0, l) d l}$.

From (66) one can see the dynamic of the spread of the forward CDS on $[0, a]$ and the proof is given in Appendix.

\section{Conclusion}

In this paper, we extend the results of Xiong and Kohlmann [5] and consider the problem of how to model the dynamics of the fair spread of the forward CDS whose recovery rate $h(L)$ depends on the loss $L$ in the jump-Heath-JarrowMorton framework. Since $L$ is known at the default time $\tilde{\tau}$, we consider the problem in the filtration $\mathbb{G}$ including $\mathbb{F}$ such that $\tilde{\tau}$ is a $\mathbb{G}$-stopping time and $L$ is $\mathscr{G}_{\tilde{\tau}}$-measurable. Because of the more general conditional density hypothesis, we introduce a new BSDEs system (45) depending on the default parameters $p_{s}(s, l), \theta_{1}(u ; s, l) \rrbracket_{u>s}$, and $\theta_{2}(u, y ; s, l) \rrbracket_{u>s}$ and the term structure parameters $C(u, T)$ and $J(u, y, T)$. By the solution of the BSDEs system (45), we can explicitly describe the term structure of the defaultable bond. As a byproduct, we can also describe the term structure of the default-free bond in the larger filtration $\mathbb{G}$. By introducing another BSDE (7) more simpler than Xiong and Kohlmann [5], we explicitly describe the dynamics of the fair spread of the forward CDS with the recovery rate $h(L)$.

\section{Appendix}

Proof of Theorem 15. (i) one can see that $M_{t}:=$ $e^{-\int_{0}^{t} r(u) d u} G_{t} B^{*}(t, T)$ is a strictly positive $(P, \mathbb{F})$-martingale, which can be rewriten as the follows:

$$
\begin{aligned}
M_{t}= & M_{0}+\int_{0}^{t} M_{u-} \xi_{1}^{d}(u)^{\prime} d W(u) \\
& +\int_{0}^{t} \int_{E} M_{u_{-}} \xi_{2}^{d}(u, y)\{\mu(d u, d y)-v(d u, d y)\},
\end{aligned}
$$

where $\xi_{1}^{d}$ is an $\mathbb{F}$-predictable process and $\xi_{2}^{d}(u, y)$ is a $\widetilde{\mathscr{P}}(\mathbb{F})$ measurable function. Since

$$
B^{*}(t, T)=M_{t} e^{\int_{0}^{t} r(u) d u} \frac{1}{G_{t}}
$$

and since

$$
\begin{aligned}
& \ln G_{t}=-\int_{0}^{t} \int_{\mathbb{R}} \frac{1}{G_{s}} p_{s}(s, l) \eta(d l) d s \\
& -\int_{0}^{t} \frac{1}{G_{u-}}\left\{1-G_{u-}\right\} \widetilde{\theta}_{1}(u)^{\prime} d W(u) \\
& +\int_{0}^{t} \int_{E} \frac{1}{G_{u-}}\left\{1-G_{u-}\right\} \widetilde{\theta}_{2}(u, y) v(d u, d y) \\
& -\frac{1}{2} \int_{0}^{t}\left\{\frac{1}{G_{u-}}-1\right\}^{2}\left\|\widetilde{\theta}_{1}(u)\right\|^{2} d u \\
& +\int_{0}^{t} \int_{E} \ln \left(1+\left(\frac{1}{G_{u-}}-1\right) \tilde{\theta}_{2}(u, y)\right) \mu(d u, d y),
\end{aligned}
$$

one can see that

$$
\begin{aligned}
\ln B^{*}( & t, T) \\
= & \ln M_{t}+\int_{0}^{t} r(u) d u-\ln G_{t} \\
= & \ln M_{0}+\int_{0}^{t}\left\{\xi_{1}^{d}(u)+\left\{\frac{1}{G_{u-}}-1\right\} \tilde{\theta}_{1}(u)\right\}^{\prime} d W(u) \\
& +\frac{1}{2} \int_{0}^{t}\left\{\left\{\frac{1}{G_{u-}}-1\right\}^{2}\left\|\widetilde{\theta}_{1}(u)\right\|^{2}-\left\|\xi_{1}^{d}(u)\right\|^{2}\right\} d u \\
& +\int_{0}^{t} \int_{E}\left\{\ln \left(1+\xi_{2}^{d}(u, y)\right)\right. \\
& -\int_{0}^{t} \int_{E}\left\{\xi_{2}^{d}(u, y)+\left\{\frac{1}{G_{u-}}-1\right\} \tilde{\theta}_{2}(u, y)\right\} \nu(d u, d y) \\
& +\int_{0}^{t} r(u) d u+\int_{0}^{t} \int_{\mathbb{R}} \frac{1}{G_{s-}} p_{s}(s, l) \eta(d l) d s ;
\end{aligned}
$$


12

Abstract and Applied Analysis

thus,

$$
\begin{aligned}
& 0=\ln B^{*}(T, T) \\
& =\ln M_{0}+\int_{0}^{T}\left\{\xi_{1}^{d}(u)+\left\{\frac{1}{G_{u-}}-1\right\} \widetilde{\theta}_{1}(u)\right\}^{\prime} d W(u) \\
& +\frac{1}{2} \int_{0}^{T}\left\{\left\{\frac{1}{G_{u-}}-1\right\}^{2}\left\|\widetilde{\theta}_{1}(u)\right\|^{2}-\left\|\xi_{1}^{d}(u)\right\|^{2}\right\} d u \\
& +\int_{0}^{T} \int_{E}\left\{\ln \left(1+\xi_{2}^{d}(u, y)\right)\right. \\
& \left.-\ln \left(1+\left(\frac{1}{G_{u-}}-1\right) \tilde{\theta}_{2}(u, y)\right)\right\} \mu(d u, d y) \\
& -\int_{0}^{T} \int_{E}\left\{\xi_{2}^{d}(u, y)+\left\{\frac{1}{G_{u-}}-1\right\} \tilde{\theta}_{2}(u, y)\right\} \nu(d u, d y) \\
& +\int_{0}^{T} \int_{\mathbb{R}} \frac{1}{G_{s-}} p_{s}(s, l) \eta(d l) d s+\int_{0}^{T} r(u) d u \\
& =\ln M_{0}+\int_{0}^{T} f(0, l) d l \\
& +\int_{0}^{T}\left\{\xi_{1}^{d}(u)-C(u, T)\right. \\
& \left.+\left\{\frac{1}{G_{u-}}-1\right\} \tilde{\theta}_{1}(u)\right\}^{\prime} d W(u) \\
& +\frac{1}{2} \int_{0}^{T}\left\{\left\{\frac{1}{G_{u-}}-1\right\}^{2}\left\|\widetilde{\theta}_{1}(u)\right\|^{2}-\left\|\xi_{1}^{d}(u)\right\|^{2}\right. \\
& \left.+\|C(u, T)\|^{2}\right\} d u \\
& +\int_{0}^{T} \int_{E}\left\{\ln \left(1+\xi_{2}^{d}(u, y)\right)\right. \\
& -\ln \left(1+\left(\frac{1}{G_{u-}}-1\right) \tilde{\theta}_{2}(u, y)\right) \\
& -J(u, y ; T)\} \mu(d u, d y) \\
& +\int_{0}^{T} \int_{E}\left\{\left\{e^{J(u, y, T)}-1\right\}-\xi_{2}^{d}(u, y)\right. \\
& \left.-\left\{\frac{1}{G_{u-}}-1\right\} \tilde{\theta}_{2}(u, y)\right\} F_{u}(d y) \\
& +\int_{0}^{T} \int_{\mathbb{R}} \frac{1}{G_{s^{-}}} p_{s}(s, l) \eta(d l) d s .
\end{aligned}
$$

If we let

$$
\begin{aligned}
& X_{t}^{d, T}=\ln M_{0}+\int_{0}^{T} f(0, l) d l \\
& +\int_{0}^{t}\left\{\xi_{1}^{d}(u)-C(u, T)\right. \\
& \left.+\left\{\frac{1}{G_{u-}}-1\right\} \tilde{\theta}_{1}(u)\right\}^{\prime} d W(u) \\
& +\frac{1}{2} \int_{0}^{t}\left\{\left\{\frac{1}{G_{u-}}-1\right\}^{2}\left\|\widetilde{\theta}_{1}(u)\right\|^{2}\right. \\
& \left.-\left\|\xi_{1}^{d}(u)\right\|^{2}+\|C(u, T)\|^{2}\right\} d u \\
& +\int_{0}^{t} \int_{E}\left\{\ln \left(1+\xi_{2}^{d}(u, y)\right)\right. \\
& -\ln \left(1+\left(\frac{1}{G_{u-}}-1\right) \widetilde{\theta}_{2}(u, y)\right) \\
& -J(u, y ; T)\} \mu(d u, d y) \\
& +\int_{0}^{T} \int_{E}\left\{\left\{e^{J(u, y, T)}-1\right\}-\xi_{2}^{d}(u, y)\right. \\
& \left.-\left\{\frac{1}{G_{u-}}-1\right\} \tilde{\theta}_{2}(u, y)\right\} F_{u}(d y) \\
& +\int_{0}^{t} \int_{\mathbb{R}} \frac{1}{G_{s-}} p_{s}(s, l) \eta(d l) d s, \\
& \xi_{1}^{d, T}(u)=\xi_{1}^{d}(u), \\
& \xi_{2}^{d, T}(u, y)=\xi_{2}^{d}(u, y),
\end{aligned}
$$

then $\left(X^{d, T}, \xi_{1}^{d, T}, \xi_{2}^{d, T}\right)$ is a solution of the BSDEs system (45).

(ii) Let $\left(X^{d, T}, \xi_{1}^{d, T}, \xi_{2}^{d, T}\right)$ be any solution of the system of BIDEs (45), and let $\Theta^{d}$ be the solution of the following SDE:

$$
\begin{aligned}
\Theta_{t}^{d}= & e^{X_{0}^{d, T}-\int_{0}^{T} f(0, l) d l} \\
& +\int_{0}^{t} \int_{\mathbb{R}} \Theta_{u-}^{d} \frac{1}{G_{s}} p_{s}(s, l) \eta(d l) d s \\
& +\int_{0}^{t} \Theta_{u-}^{d}\left\{r(u)+\left\|\left\{\frac{1}{G_{u-}}-1\right\} \widetilde{\theta}_{1}(u)+\frac{1}{2} \xi_{1}^{d, T}(u)\right\|^{2}\right. \\
& \left.\quad-\frac{1}{4}\left\|\xi_{1}^{d, T}(u)\right\|^{2}\right\} d u \\
& +\int_{0}^{t} \int_{E} \Theta_{u-}^{d}\left\{\frac{1}{G_{u-}}-1\right\} \widetilde{\theta}_{2}(u, y)
\end{aligned}
$$




$$
\begin{array}{r}
\times\left\{\frac{1+\xi_{2}^{d, T}(u, y)}{1-\left\{1 / G_{u-}-1\right\} \widetilde{\theta}_{2}(u, y)}-1\right\} F_{u}(d y) d u \\
+\int_{0}^{t} \Theta_{u-}^{d}\left\{\left\{\frac{1}{G_{u-}}-1\right\} \widetilde{\theta}_{1}(u)+\xi_{1}^{d, T}(u)\right\}^{\prime} d W(u) \\
+\int_{0}^{t} \int_{E} \Theta_{u-}^{d}\left\{\frac{1+\xi_{2}^{d, T}(u, y)}{1-\left\{1 / G_{u-}-1\right\} \widetilde{\theta}_{2}(u, y)}\right. \\
-1\}\{\mu(d u, d y)-v(d u, d y)\} .
\end{array}
$$

One can see from Itô's formula that $K_{t}:=\Theta_{t}^{d} e^{-\int_{0}^{t} r(u) d u} G_{t}$ satisfies the SDE:

$$
\begin{aligned}
K_{t}= & e^{X_{0}^{d, T}-\int_{0}^{T} f(0, l) d l}+\int_{0}^{t} K_{u-} \xi_{1}^{d, T}(u)^{\prime} d W(u) \\
& +\int_{0}^{t} \int_{E} K_{u-} \xi_{2}^{d, T}(u, y)\{\mu(d u, d y)-v(d u, d y)\} .
\end{aligned}
$$

Thus, the process $K_{t}$ is a $(P, \mathbb{G})$ uniformly integrable martingale. Furthermore, once again from Itô's formula, one can see that

$$
\begin{aligned}
\ln \Theta_{T}^{d}= & X_{0}^{d, T}-\int_{0}^{T} f(0, l) d l \\
+ & \int_{0}^{T}\left\{\xi_{1}^{d, T}(u)-C(u, T)\right. \\
& \left.+\left\{\frac{1}{G_{u-}}-1\right\} \widetilde{\theta}_{1}(u)\right\}^{\prime} d W(u) \\
+ & \frac{1}{2} \int_{0}^{T}\left\{\left\{\frac{1}{G_{u-}}-1\right\}^{2}\left\|\widetilde{\theta}_{1}(u)\right\|^{2}-\left\|\xi_{1}^{d, T}(u)\right\|^{2}\right. \\
& \left.+\|C(u, T)\|^{2}\right\} d u \\
+ & \int_{0}^{T} \int_{E}\left\{\ln \left(1+\xi_{2}^{d, T}(u, y)\right)\right. \\
& \quad-\ln \left(1+\left(\frac{1}{G_{u-}}-1\right) \widetilde{\theta}_{2}(u, y)\right) \\
& \quad-J(u, y ; T)\} \mu(d u, d y) \\
+ & \int_{0}^{T} \int_{E}\left\{\left\{e^{J(u, y, T)}-1\right\}-\xi_{2}^{d, T}(u, y)\right.
\end{aligned}
$$

$$
\begin{aligned}
&\left.-\left\{\frac{1}{G_{u-}}-1\right\} \widetilde{\theta}_{2}(u, y)\right\} F_{u}(d y) \\
&+\int_{0}^{T} \int_{\mathbb{R}} \frac{1}{G_{s-}} p_{s}(s, l) \eta(d l) d s \\
&=X_{T}^{d, T}=0,
\end{aligned}
$$

and thus $\Theta_{T}^{d}=1$. Therefore,

$$
\begin{aligned}
\Theta_{t}^{d} e^{-\int_{0}^{t} r(u) d u} G_{t} & =K_{t}=E\left[K_{T} \mid \mathscr{F}_{t}\right] \\
& =E\left[e^{-\int_{0}^{T} r(u) d u} G_{T} \mid \mathscr{F}_{t}\right],
\end{aligned}
$$

which implies

$$
\Theta_{t}^{d}=E\left[e^{-\int_{t}^{T} r(u) d u} \frac{G_{T}}{G_{t}} \mid \mathscr{F}_{t}\right]=B^{*}(t, T), \quad \text { a.s. }
$$

Proof of Theorem 19. (i) Let $M_{t}:=B(t, T) e^{-\int_{0}^{t} r(u) d u}$; one can see that $M$ is a strictly positive $(P, \mathbb{G})$-martingale; according to Corollary 12, one can see that $M$ can be represented into the following:

$$
\begin{aligned}
M_{t}= & M_{0}+\int_{0}^{t} M_{u-} \xi_{1}(u)^{\prime} d W^{\mathbb{G}}(u) \\
& +\int_{0}^{t} \int_{E} M_{u^{-}} \xi_{2}(u, y)\left\{\mu(d u, d y)-\nu^{\mathbb{G}}(d u, d y)\right\} \\
& +M_{\tilde{\tau}-} h(\tilde{\tau} ; \widetilde{\tau}, L) \rrbracket_{t \geq \tilde{\tau}} \\
& -\int_{0}^{t \wedge \tilde{\tau}} \int_{R} M_{s-} h(s ; s, l) \frac{1}{G_{s-}} p_{s}(s, l) \eta(d l) d s,
\end{aligned}
$$

where $\xi_{1}(u)$ is an $\mathbb{R}^{n}$-valued $\mathbb{G}$-predictable process, $\xi_{2}(u, y)$ is a $\widetilde{P}(\mathbb{G})$-measurable function with $\xi_{2}(u, y)>-1$, and $h(s ; s, l)$ is a $\mathcal{O}(\mathbb{F}) \otimes \mathscr{B}\left(\mathbb{R}^{+}\right) \otimes \mathscr{B}(\mathbb{R})$-measurable function $h(s ; s, l)>-1$. It is notable that $\xi_{1}, \xi_{2}$, and $h$ may depend on $T$. Thus,

$$
\begin{aligned}
B(t, T)= & M_{t} e^{\int_{0}^{t} r(u) d u} \\
= & M_{0}+\int_{0}^{t} B(s-, T) r(s) d s \\
& +\int_{0}^{t} B(u-, T) \xi_{1}(u)^{\prime} d W^{\mathbb{G}}(u) \\
& +\int_{0}^{t} \int_{E} B(u-, T) \xi_{2}(u, y)\left\{\mu(d u, d y)-v^{\mathbb{G}}(d u, d y)\right\} \\
& +B(\widetilde{\tau}-, T) h(\tilde{\tau} ; \widetilde{\tau}, L) \mathbb{\square}_{t \geq \tilde{\tau}} \\
& -\int_{0}^{t \wedge \tau} \int_{R} B(s-, T) h(s ; s, l) \frac{1}{G_{s-}} p_{s}(s, l) \eta(d l) d s,
\end{aligned}
$$


which implies

$$
\begin{aligned}
\ln B(t, T)= & \ln M_{0}+\int_{0}^{t} r(s) d s \\
& +\int_{0}^{t} \xi_{1}(u)^{\prime} d W^{\mathbb{G}}(u)-\frac{1}{2} \int_{0}^{t}\left\|\xi_{1}(u)\right\|^{2} d u \\
& +\int_{0}^{t} \int_{E} \ln \left\{1+\xi_{2}(u, y)\right\} \mu(d u, d y) \\
& -\int_{0}^{t} \int_{E} \xi_{2}(u, y) \nu^{\mathbb{G}}(d u, d y)+\ln \{1+h(\tilde{\tau} ; \widetilde{\tau}, L)\} \square_{t \geq \tau} \\
& -\int_{0}^{t \wedge \tilde{\tau}} \int_{R} h(s ; s, l) \frac{1}{G_{s-}} p_{s}(s, l) \eta(d l) d s .
\end{aligned}
$$

Since $B(T, T)=1$, then

$$
\begin{aligned}
0= & \ln M_{0}+\int_{0}^{T} r(s) d s \\
& +\int_{0}^{T} \xi_{1}(u)^{\prime} d W^{\mathbb{G}}(u)-\frac{1}{2} \int_{0}^{T}\left\|\xi_{1}(u)\right\|^{2} d u \\
& +\int_{0}^{T} \int_{E} \ln \left\{1+\xi_{2}(u, y)\right\} \mu(d u, d y) \\
& -\int_{0}^{T} \int_{E} \xi_{2}(u, y) \nu^{\mathbb{G}}(d u, d y) \\
& +\ln \{1+h(\tilde{\tau} ; \tilde{\tau}, L)\} \square_{T \geq \tilde{\tau}} \\
& -\int_{0}^{T \wedge \tilde{\tau}} \int_{R} h(s ; s, l) \frac{1}{G_{s-}} p_{s}(s, l) \eta(d l) d s,
\end{aligned}
$$

and it follows from (42) that

$$
\begin{aligned}
0= & \ln M_{0}+\int_{0}^{T} f(0, l) d l \\
& +\int_{0}^{T}\left\{\xi_{1}(u)-C(u, T)\right\}^{\prime} d W^{\mathbb{G}}(u) \\
& +\int_{0}^{T}\left\{-\frac{1}{2}\left\|\xi_{1}(u)\right\|^{2}+\frac{1}{2}\|C(u, T)\|^{2}+C(u, T)^{\prime}\right. \\
& \left.\times\left\{\left\{\frac{1}{G_{u-}}-1\right\} \widetilde{\theta}_{1}(u) \mathbb{q}_{u \leq \tilde{\tau}}-\theta_{1}(u ; \widetilde{\tau}, L) \mathbb{\square}_{u>\tau}\right\}\right\} d u
\end{aligned}
$$

$$
\begin{gathered}
+\int_{0}^{T} \int_{E}\left\{\ln \left\{1+\xi_{2}(u, y)\right\}-J(u, y ; T)\right\} \mu(d u, d y) \\
+\int_{0}^{T} \int_{E}\left\{\left\{e^{J(u, y, T)}-1\right\}-\xi_{2}(u, y)\right. \\
\times\left\{1-\left\{\frac{1}{G_{u-}}-1\right\} \widetilde{\theta}_{2}(u, y) \rrbracket_{u \leq \tau}\right. \\
\left.\left.+\theta_{2}(u, y ; \widetilde{\tau}, L) \rrbracket_{u>\tau}\right\}\right\} F_{u}(d y) d u \\
+\ln \{1+h(\tilde{\tau} ; \tilde{\tau}, L)\} \square_{T \geq \tau} \\
-\int_{0}^{T \wedge \tilde{\tau}} \int_{R} h(s ; s, l) \frac{1}{G_{s-}} p_{s}(s, l) \eta(d l) d s ;
\end{gathered}
$$

if we let

$$
\begin{aligned}
& X_{t}^{T}=\ln M_{0}+\int_{0}^{t}\left\{\xi_{1}(u)-C(u, T)\right\}^{\prime} d W^{\mathbb{G}}(u) \\
& +\int_{0}^{t}\left\{-\frac{1}{2}\left\|\xi_{1}(u)\right\|^{2}+\frac{1}{2}\|C(u, T)\|^{2}+C(u, T)^{\prime}\right. \\
& \left.\times\left\{\left\{\frac{1}{G_{u-}}-1\right\} \tilde{\theta}_{1}(u) \rrbracket_{u \leq \tau}-\theta_{1}(u ; \tilde{\tau}, L) \rrbracket_{u>\tau}\right\}\right\} d u \\
& +\int_{0}^{t} \int_{E}\left\{\ln \left\{1+\xi_{2}(u, y)\right\}-J(u, y ; T)\right\} \mu(d u, d y) \\
& +\int_{0}^{t} \int_{E}\left\{\left\{e^{J(u, y, T)}-1\right\}-\xi_{2}(u, y)\right. \\
& \times\left\{1-\left\{\frac{1}{G_{u-}}-1\right\} \widetilde{\theta}_{2}(u, y) \rrbracket_{u \leq \tau}\right. \\
& \left.\left.+\theta_{2}(u, y ; \widetilde{\tau}, L) \rrbracket_{u>\tau}\right\}\right\} F_{u}(d y) d u \\
& +\ln \{1+h(\tilde{\tau} ; \tilde{\tau}, L)\} \square_{t \geq \tilde{\tau}} \\
& -\int_{0}^{t \wedge \tilde{\tau}} \int_{R} h(s ; s, l) \frac{1}{G_{s-}} p_{s}(s, l) \eta(d l) d s, \\
& \xi_{1}^{T}(u)=\xi_{1}(u), \\
& \xi_{2}^{T}(u, y)=\xi_{2}(u, y), \\
& h^{T}(u ; s, l)=h(u ; s, l),
\end{aligned}
$$

then $\left(X^{T}, \xi_{1}^{T}, \xi_{2}^{T}, h^{T}\right)$ is a solution of the BSDEs system (54). 
(ii) Let $\left(X^{T}, \xi_{1}^{T}, \xi_{2}^{T}, h^{T}\right)$ be any solution of the system of BSDEs (54), and let $\Theta$ be the solution of the following SDE:

$$
\begin{aligned}
\Theta_{t}= & e^{X_{0}^{T}-\int_{0}^{T} f(0, l) d l}+\int_{0}^{t} \Theta_{u_{-}} r(u) d u \\
& +\int_{0}^{t} \Theta_{u-} \xi_{1}^{T}(u)^{\prime} d W^{\mathbb{G}}(u) \\
& +\int_{0}^{t} \int_{E} \Theta_{u_{-}} \xi_{2}^{T}(u, y)\left\{\mu(d u, d y)-\nu^{\mathbb{G}}(d u, d y)\right\} \\
& +\Theta_{\widetilde{\tau}-} h^{T}(\tilde{\tau} ; \widetilde{\tau}, L) \square_{t \geq \tilde{\tau}} \\
& -\int_{0}^{t \wedge \tilde{\tau}} \int_{R} \Theta_{s-} h^{T}(s ; s, l) \frac{1}{G_{s-}} p_{s}(s, l) \eta(d l) d s .
\end{aligned}
$$

One can see that the process $\Theta_{t} e^{-\int_{0}^{t} r(u) d u}$ is a $(P, \mathbb{G})$ uniformly integrable martingale, and from Itô's formula, we see that

$$
\begin{aligned}
\ln \Theta_{T}= & X_{0}^{T}-\int_{0}^{T} f(0, l) d l+\int_{0}^{T} r(s) d s \\
& +\int_{0}^{T} \xi_{1}^{T}(u)^{\prime} d W^{\mathbb{G}}(u)-\frac{1}{2} \int_{0}^{t}\left\|\xi_{1}^{T}(u)\right\|^{2} d u \\
& +\int_{0}^{T} \int_{E} \ln \left\{1+\xi_{2}^{T}(u, y)\right\} \mu(d u, d y) \\
& -\int_{0}^{t} \int_{E} \xi_{2}^{T}(u, y) \nu^{\mathbb{G}}(d u, d y)+\ln \left\{1+h^{T}(\tilde{\tau} ; \tilde{\tau}, L)\right\} \mathbb{\square}_{T \geq \tilde{\tau}} \\
& -\int_{0}^{T \wedge \tau} \int_{R} h^{T}(s ; s, l) \frac{1}{G_{s-}} p_{s}(s, l) \eta(d l) d s \\
= & X_{0}^{T}+\int_{0}^{T}\left\{\frac{1}{2}\|C(u, T)\|^{2}\right. \\
& +\int_{0}^{T} \xi_{1}^{T}(u)^{\prime} d W^{\mathbb{G}}(u)-\frac{1}{2} \int_{0}^{t}\left\|\xi_{1}^{T}(u)\right\|^{2} d u \\
& -\int_{0}^{T} C(u, T)^{\prime} d W(u)-\xi_{0}^{T}\left\{\int_{E} J(u, y, T) \mu(d u, d y)\right. \\
& \left.\left.+\int_{E}^{J(u, y, T)}-1\right\} F_{u}(d y)\right\} d u
\end{aligned}
$$

$$
\begin{aligned}
& -\int_{0}^{T \wedge \tilde{\tau}} \int_{R} h^{T}(s ; s, l) \frac{1}{G_{s-}} p_{s}(s, l) \eta(d l) d s \\
= & X_{T}^{T}=0,
\end{aligned}
$$

and thus $\Theta_{T}=1$ and

$$
\Theta_{t} e^{-\int_{0}^{t} r(u) d u}=E\left[e^{-\int_{0}^{T} r(u) d u} \mid \mathscr{G}_{t}\right],
$$

from which one can see that $\Theta_{t}=E\left[e^{-\int_{t}^{T} r(u) d u} \mid \mathscr{G}_{t}\right]=$ $B(t, T)$, a.s., which completes the proof.

$$
\begin{aligned}
& \text { Proof of Theorem 25. Since } B^{*}\left(t, T_{k}\right) \\
& E\left(e^{-\int_{t}^{T} r(u) d u}\left(G_{T_{k}} / G_{t}\right) \quad \mid \quad \mathscr{F}_{t}\right) \text {, one can see that } \\
& M_{t}^{k}:=e^{-\int_{0}^{t} r(u) d u} G_{t} B^{*}\left(t, T_{k}\right) \text { is a strictly positive }(P, \mathbb{F})- \\
& \text { martingale, which can be rewriten as the following: }
\end{aligned}
$$

$$
\begin{aligned}
M_{t}^{k}= & M_{0}^{k}+\int_{0}^{t} M_{u-}^{k} \xi_{1}^{d, T_{k}}(u)^{\prime} d W(u) \\
& +\int_{0}^{t} \int_{E} M_{u-}^{k} \xi_{2}^{d, T_{k}}(u, y)\{\mu(d u, d y)-v(d u, d y)\}
\end{aligned}
$$

Let $M_{t}=\sum_{k=1}^{n} M_{t}^{k}$; then,

$$
\begin{aligned}
M_{t}= & M_{0}+\int_{0}^{t} M_{u-}\left(\frac{\sum_{k=1}^{n} M_{u-}^{k} \xi_{1}^{d, T_{k}}(u)}{\sum_{k=1}^{n} M_{u-}^{k}}\right)^{\prime} d W(u) \\
+ & \int_{0}^{t} \int_{E} M_{u-} \\
& \times \frac{\sum_{k=1}^{n} M_{u-}^{k} \xi_{2}^{d, T_{k}}(u, y)}{\sum_{k=1}^{n} M_{u-}^{k}}\{\mu(d u, d y)-v(d u, d y)\},
\end{aligned}
$$

and thus, for $t<a$,

$$
\begin{aligned}
\mathscr{R}_{t} & =\frac{D(t, a, b)}{\sum_{k=1}^{n} B^{*}\left(t, T_{k}\right)} \rrbracket_{t<\tilde{\tau}} \\
& =\frac{Y_{t}}{\sum_{k=1}^{n} M_{t}^{k}} \rrbracket_{t<\tau}=\frac{Y_{t}}{M_{t}} \rrbracket_{t<\tilde{\tau}} \\
& =\mathscr{R}_{t}^{*} \rrbracket_{t<\tilde{\tau}} .
\end{aligned}
$$

From Itô's formula, one can see that

$$
\begin{aligned}
\mathscr{R}_{t}^{*}= & \frac{Y_{0}}{M_{0}}+\int_{0}^{t} \frac{1}{M_{u-}} d Y_{u} \\
& -\int_{0}^{t} \frac{Y_{u-}}{M_{u-}^{2}} d M_{u}+\int_{0}^{t} \frac{Y_{u-}}{M_{u-}^{3}} d\langle M, M\rangle_{u}
\end{aligned}
$$




$$
\begin{aligned}
& -\int_{0}^{t} \frac{1}{M_{u-}^{2}} d\langle Y, M\rangle_{u} \\
& +\int_{0}^{t} \int_{E} \mathscr{R}_{u-}^{*}\left\{\frac{1+\zeta_{2}(u, y)}{1+\sum_{k=1}^{n} M_{u-}^{k} \xi_{2}^{d, T_{k}}(u, y) / \sum_{k=1}^{n} M_{u-}^{k}}\right. \\
& -1+\frac{\sum_{k=1}^{n} M_{u-}^{k} \xi_{2}^{d, T_{k}}(u, y)}{\sum_{k=1}^{n} M_{u-}^{k}} \\
& \left.-\zeta_{2}(u, y)\right\} \mu(d u, d y) \\
& =\frac{Y_{0}}{M_{0}}+\int_{0}^{t} \mathscr{R}_{u-}^{*} \zeta_{1}(u)^{\prime} d W(u) \\
& +\int_{0}^{t} \mathscr{R}_{u-}^{*} \zeta_{2}(u, y)\{\mu(d u, d y)-v(d u, d y)\} \\
& -\int_{0}^{t} \mathscr{R}_{u-}^{*}\left(\frac{\sum_{k=1}^{n} M_{u-}^{k} \xi_{1}^{d, T_{k}}(u)}{\sum_{k=1}^{n} M_{u-}^{k}}\right)^{\prime} d W(u) \\
& -\int_{0}^{t} \int_{E} \mathscr{R}_{u-}^{*} \\
& \times \frac{\sum_{k=1}^{n} M_{u_{-}}^{k} \xi_{2}^{d, T_{k}}(u, y)}{\sum_{k=1}^{n} M_{u^{-}}^{k}}\{\mu(d u, d y)-\nu(d u, d y)\} \\
& +\int_{0}^{t} \mathscr{R}_{u-}^{*}\left\|\frac{\sum_{k=1}^{n} M_{u-}^{k} \xi_{1}^{d, T_{k}}(u)}{\sum_{k=1}^{n} M_{u-}^{k}}\right\|^{2} d u \\
& -\int_{0}^{t} \mathscr{R}_{u-}^{*} \frac{\sum_{k=1}^{n} M_{u-}^{k} \xi_{1}^{d, T_{k}}(u)^{\prime}}{\sum_{k=1}^{n} M_{u-}^{k}} \zeta_{1}(u) d u \\
& +\int_{0}^{t} \int_{E} \mathscr{R}_{u-}^{*}\left\{\frac{1+\zeta_{2}(u, y)}{1+\sum_{k=1}^{n} M_{u^{-}}^{k} \xi_{2}^{d, T_{k}}(u, y) / \sum_{k=1}^{n} M_{u^{-}}^{k}}\right. \\
& -1+\frac{\sum_{k=1}^{n} M_{u-}^{k} \xi_{2}^{d, T_{k}}(u, y)}{\sum_{k=1}^{n} M_{u-}^{k}} \\
& \left.-\zeta_{2}(u, y)\right\} \mu(d u, d y) \\
& =\frac{Y_{0}}{M_{0}}+\int_{0}^{t} \mathscr{R}_{u-}^{*} \zeta_{1}(u)^{\prime} d W(u) \\
& -\int_{0}^{t} \mathscr{R}_{u-}^{*} \zeta_{2}(u, y) \nu(d u, d y) \\
& -\int_{0}^{t} \mathscr{R}_{u-}^{*}\left(\frac{\sum_{k=1}^{n} M_{u-}^{k} \xi_{1}^{d, T_{k}}(u)}{\sum_{k=1}^{n} M_{u-}^{k}}\right)^{\prime} d W(u) \\
& +\int_{0}^{t} \int_{E} \mathscr{R}_{u-}^{*} \frac{\sum_{k=1}^{n} M_{u-}^{k} \xi_{2}^{d, T_{k}}(u, y)}{\sum_{k=1}^{n} M_{u-}^{k}} \nu(d u, d y) \\
& +\int_{0}^{t} \mathscr{R}_{u^{-}}^{*}\left\|\frac{\sum_{k=1}^{n} M_{u-}^{k} \xi_{1}^{d, T_{k}}(u)}{\sum_{k=1}^{n} M_{u-}^{k}}\right\|^{2} d u
\end{aligned}
$$

$$
\begin{gathered}
-\int_{0}^{t} \mathscr{R}_{u-}^{*} \frac{\sum_{k=1}^{n} M_{u-}^{k} \xi_{1}^{d, T_{k}}(u)^{\prime}}{\sum_{k=1}^{n} M_{u-}^{k}} \zeta_{1}(u) d u \\
+\int_{0}^{t} \int_{E} \mathscr{R}_{u-}^{*}\left\{\frac{1+\zeta_{2}(u, y)}{1+\sum_{k=1}^{n} M_{u-}^{k} \xi_{2}^{d, T_{k}}(u, y) / \sum_{k=1}^{n} M_{u-}^{k}}\right. \\
-1\} \mu(d u, d y),
\end{gathered}
$$

which implies

$$
\begin{aligned}
\mathscr{R}_{t}^{*}= & \frac{Y_{0}}{M_{0}}-\int_{0}^{t} \mathscr{R}_{u-}^{*}\left\{\frac{\sum_{k=1}^{n} M_{u-}^{k} \xi_{1}^{d, T_{k}}(u)}{\sum_{k=1}^{n} M_{u-}^{k}}\right. \\
& \left.-\zeta_{1}(u)\right\}^{\prime} d W(u) \\
+ & \int_{0}^{t} \int_{E} \mathscr{R}_{u-}^{*}\left\{\frac{1+\zeta_{2}(u, y)}{1+\sum_{k=1}^{n} M_{u-}^{k} \xi_{2}^{d, T_{k}}(u, y) / \sum_{k=1}^{n} M_{u^{-}}^{k}}\right. \\
+ & \int_{0}^{t} \mathscr{R}_{u-}^{*} \frac{\sum_{k=1}^{n} M_{u-}^{k} \xi_{1}^{d, T_{k}}(u)^{\prime}}{\sum_{k=1}^{n} M_{u-}^{k}} \\
& \times\left\{\frac{\sum_{k=1}^{n} M_{u-}^{k} \xi_{1}^{d, T_{k}}(u)}{\sum_{k=1}^{n} M_{u-}^{k}}-\zeta_{1}(u)\right\} d u \\
+ & \int_{0}^{t} \int_{E} \mathscr{R}_{u-}^{*}\left\{\frac{\sum_{k=1}^{n} M_{u-}^{k} \xi_{2}^{d, T_{k}}(u, y)}{\sum_{k=1}^{n} M_{u-}^{k}}-\zeta_{2}(u, y)\right\} \\
& \times \frac{\sum_{k=1}^{n} M_{u-}^{k} \xi_{2}^{d, T_{k}}(u, y) / \sum_{k=1}^{n} M_{u-}^{k}}{1+\sum_{k=1}^{n} M_{u-}^{k} \xi_{2}^{d, T_{k}}(u, y) / \sum_{k=1}^{n} M_{u-}^{k}} \\
& \times F_{u}(d y) d u,
\end{aligned}
$$

which is (66).

\section{Conflict of Interests}

The authors declare that there is no conflict of interests regarding the publication of this paper.

\section{Acknowledgments}

This work is supported by National Natural Science Foundation of China (no. 11171215), National Natural Science foundation of Shanghai (no. 13ZR1422000), and Yang Cai Project (no. YC-XK-13106). 


\section{References}

[1] D. Brigo, "Market models for CDS options and callable floaters," Risk Magazine, reprinted in Derivatives Trading and Option Pricing, N. Dunbar, Ed., Risk Books, London, UK, 2005.

[2] D. Brigo, "Constant maturity CDS valuation with market models," Risk Magazine, 2006.

[3] B. Li and M. Rutkowski, "Market models of forward CDS spreads," in Stochastic Analysis with Financial Applications, vol. 65 of Progress in Probability, pp. 361-411, 2011.

[4] M. Rutkowski and A. Armstrong, "Valuation of credit default swaptions and credit default index swaptions," International Journal of Theoretical and Applied Finance, vol. 12, no. 7, pp. 1027-1053, 2009.

[5] D. Xiong and M. Kohlmann, "Modeling the forward CDS spreads with jumps," Stochastic Analysis and Applications, vol. 30, no. 3, pp. 375-402, 2012.

[6] T. Björk, Y. Kabanov, and W. Runggaldier, "Bond market structure in the presence of marked point processes," Mathematical Finance, vol. 7, no. 2, pp. 211-239, 1997.

[7] J. Jacod, "Grossissement initial, Hypothèse $(\mathrm{H})$ et théorème de Girsanov," in Séminaire de Calcul Stochastique 1982/83, vol. 1118 of Lecture Notes in Mathematics, Springer, 1987.

[8] J. Amendinger, Initial enlargement of filtrations and additional information in financial markets [Ph.D. thesis], Technischen Universität Berlin, 1999.

[9] G. Callegaro, M. Jeanblanc, and B. Zargari, "Carthaginian enlargement of filtrations," ESAIM: Probability and Statistics, vol. 17, pp. 550-566, 2013.

[10] C. Dellacherie and P. A. Meyer, A Propos des Réesultats de Yor Sur le Grossissement des Tribus Séminaire de Probabilités, 1978.

[11] N. El Karoui, M. Jeanblanc, and Y. Jiao, "What happens after a default: the conditional density approach," Stochastic Processes and Their Applications, vol. 120, no. 7, pp. 1011-1032, 2010.

[12] M. Jeanblanc and Y. Le Cam, "Progressive enlargement of filtrations with initial times," Stochastic Processes and Their Applications, vol. 119, no. 8, pp. 2523-2543, 2009.

[13] M. Jeanblanc and S. Song, "An explicit model of default time with given survival probability," Stochastic Processes and Their Applications, vol. 121, no. 8, pp. 1678-1704, 2011.

[14] M. Jeanblanc and S. Song, "Martingale representation property in progressively enlarged filtrations," Working Paper, 2012.

[15] J. Jacod and A. N. Shiryaev, Limit Theorems for Stochastic Processes, vol. 288, Springer, Berlin, Germany, 1987.

[16] Y. Kchia, M. Larsson, and P. Protter, "On progressive filtration expansions with a process: applications to insider trading," In press.

[17] Y. Kchia, M. Larsson, and P. Protter, "Linking progressive and initial filtration expansions," in Malliavin Calculus and Stochastic Analysis, vol. 34 of Springer Proceedings in Mathematics \& Statistics, pp. 469-487, Springer, 2013.

[18] T. Jeulin, Semi-Martingales et Grossissement d'une Filtration, vol. 833 of Lecture Notes in Mathematics, Springer, New York, NY, USA, 1980.

[19] M. Jeanblanc, M. Yor, and M. Chesney, Mathematical Methods for Financial Markets, Springer, London, UK, 2009.

[20] H. Pham, "Stochastic control under progressive enlargement of filtrations and applications to multiple defaults risk management," Stochastic Processes and their Applications, vol. 120, no. 9, pp. 1795-1820, 2010.
[21] K. Tian, D. Xiong, and Z. Ye, "The martingale representation in a progressive enlargement of a filtration with jumps," 2013, http://arxiv.org/pdf/1301.1119vl.pdf.

[22] D. Xiong and M. Kohlmann, "Defaultable bond markets with jumps," Stochastic Analysis and Applications, vol. 30, no. 2, pp. 285-321, 2012.

[23] D. Lando, "On cox processes and credit risky securities," Review of Derivatives Research, vol. 2, no. 2-3, pp. 99-120, 1998. 


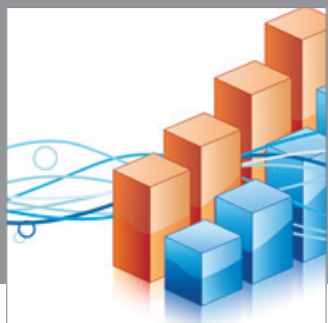

Advances in

Operations Research

mansans

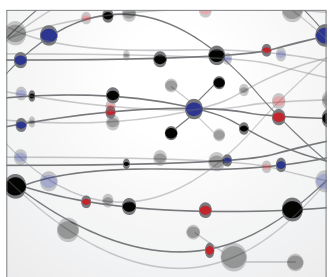

The Scientific World Journal
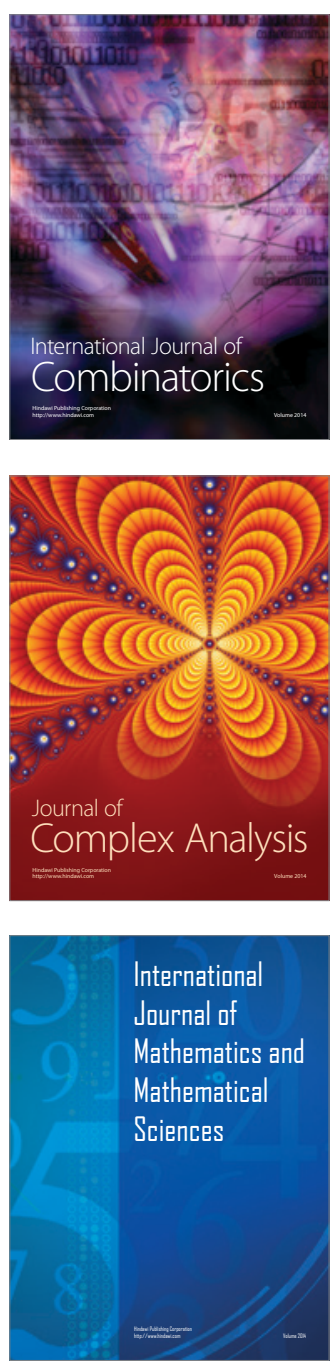
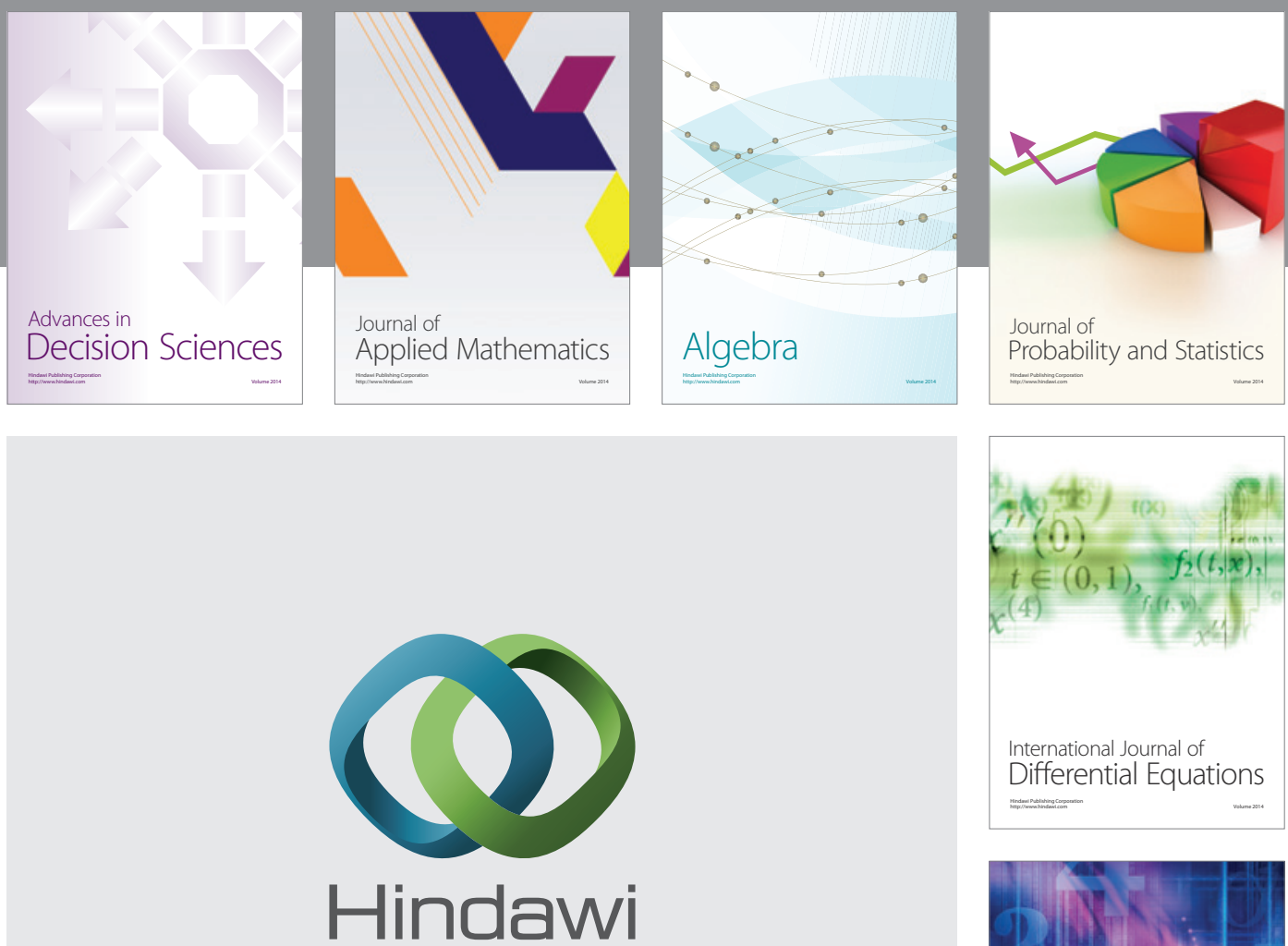

Submit your manuscripts at http://www.hindawi.com
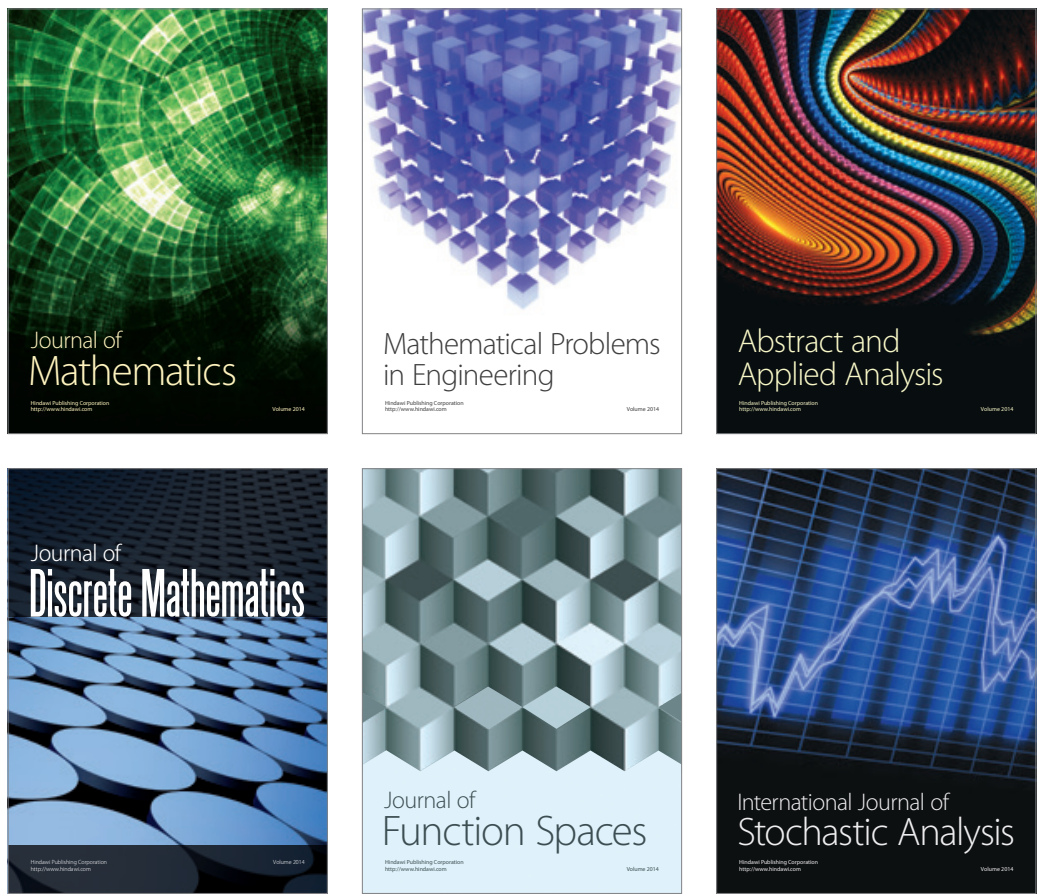

Journal of

Function Spaces

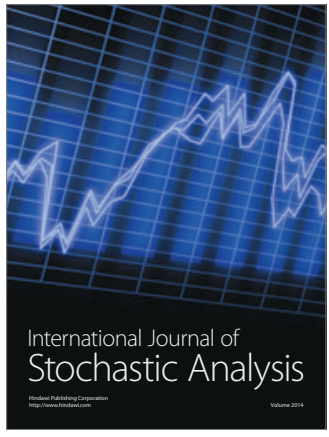

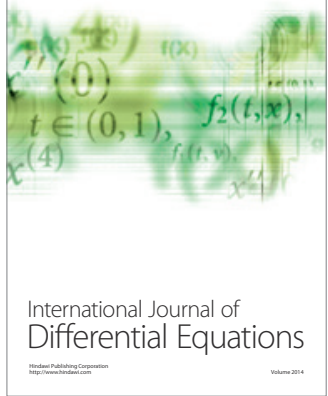
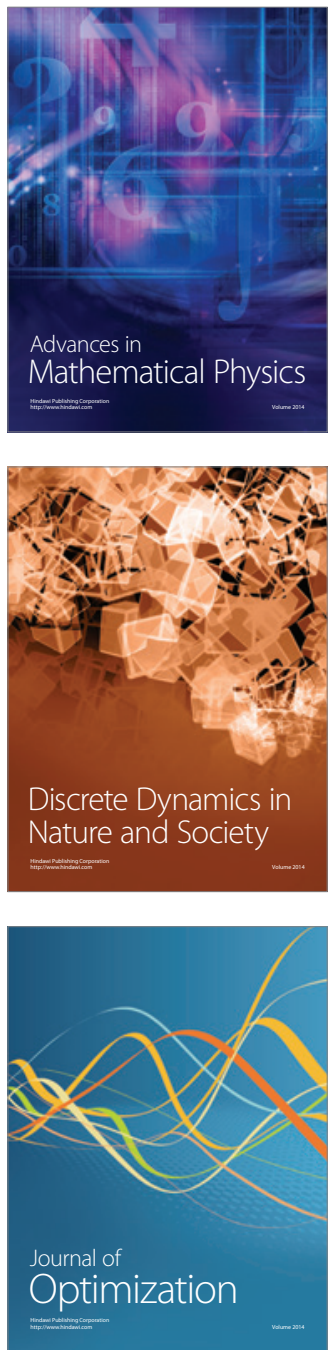